\title{
Periodic bouncing modes for two uniformly magnetized spheres. I. Trajectories
}

Cite as: Chaos 30, 013146 (2020); https://doi.org/10.1063/1.5125924

Submitted: 27 August 2019 . Accepted: 30 December 2019 . Published Online: 28 January 2020

Boyd F. Edwards (D), Bo A. Johnson (D), and John M. Edwards (D)

\section{COLLECTIONS}

F This paper was selected as Featured
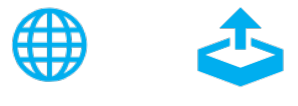

\section{ARTICLES YOU MAY BE INTERESTED IN}

Periodic bouncing modes for two uniformly magnetized spheres. II. Scaling

Chaos: An Interdisciplinary Journal of Nonlinear Science 30, 013131 (2020); https:// doi.org/10.1063/1.5125925

Coarse-scale PDEs from fine-scale observations via machine learning

Chaos: An Interdisciplinary Journal of Nonlinear Science 30, 013141 (2020); https:// doi.org/10.1063/1.5126869

Identification of Colombian coffee price dynamics

Chaos: An Interdisciplinary Journal of Nonlinear Science 30, 013145 (2020); https://

doi.org/10.1063/1.5119857

\section{Scilight}




\title{
Periodic bouncing modes for two uniformly magnetized spheres. I. Trajectories
}

\author{
Cite as: Chaos 30, 013146 (2020); doi: 10.1063/1.5125924 \\ Submitted: 27 August 2019 . Accepted: 30 December 2019 . \\ Published Online: 28 January 2020
}

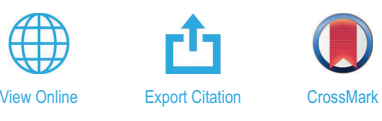

Boyd F. Edwards, ', a) (D) Bo A. Johnson, ? (D) and John M. Edwards ${ }^{2}$ (D)

\author{
AFFILIATIONS \\ ${ }^{1}$ Department of Physics, Utah State University, Logan, Utah 84322, USA \\ ${ }^{2}$ Department of Computer Science, Utah State University, Logan, Utah 84322, USA
}

a) Author to whom correspondence should be addressed: boyd.edwards@usu.edu

\begin{abstract}
We consider a uniformly magnetized sphere that moves without friction in a plane in response to the field of a second, identical, fixed sphere, making elastic hard-sphere collisions with this sphere. We seek periodic solutions to the associated nonlinear equations of motion. We find closed-form mathematical solutions for small-amplitude modes and use these to characterize and validate our large-amplitude modes, which we find numerically. Our Runge-Kutta integration approach allows us to find 1243 distinct periodic modes with the free sphere located initially at its stable equilibrium position. Each of these modes bifurcates from the finite-amplitude radial bouncing mode with infinitesimalamplitude angular motion and supports a family of states with increasing amounts of angular motion. These states offer a rich variety of behaviors and beautiful, symmetric trajectories, including states with up to 157 collisions and 580 angular oscillations per period.
\end{abstract}

Published under license by AIP Publishing. https://doi.org/10.1063/1.5125924

A vibrant online learning community shares information about building beautiful sculptures from collections of small neodymium magnet spheres, with YouTube tutorial videos attracting over a hundred million views. ${ }^{1,2}$ These spheres offer engaging hands-on exposure to principles of magnetism and are used both in and out of the classroom to teach principles of mathematics, physics, chemistry, biology, and engineering. ${ }^{3} \mathrm{We}$ showed recently that the forces and torques between two uniformly magnetized spheres are identical to the forces and torques between two point magnetic dipoles. In this paper, we exploit this equivalence to study the conservative nonlinear dynamics of a uniformly magnetized sphere subject to the magnetic forces and torques produced by a second, fixed, uniformly magnetized sphere, assuming frictionless hard-sphere elastic collisions between them. Our search for periodic states uncovers a wide variety of periodic modes, some of which are highly complex and beautiful.

\section{INTRODUCTION}

Scientific investigations of the behavior of collections of spherical magnets include studies of chain, ring, and tube formation, ${ }^{4-6}$ a continuum model for magnet chain energy, ${ }^{7}$ mechanical properties of chains and cylinders, ${ }^{8}$ stable defects along chains and rings, ${ }^{9}$ eigenmodes for lateral oscillations of a straight chain, ${ }^{10}$ stability of vertical chains, ${ }^{11}$ and radiation damping for two magnets oscillating in each other's fields. ${ }^{12}$ These studies emphasize the energetics and stability of symmetric assemblies of many spherical magnets.

These investigations treat spherical magnets as uniformly magnetized spheres, meaning that the net dipole moment per unit volume, called the magnetization, has uniform magnitude and direction throughout the sphere. Such permanent ferromagnetism arises primarily from the alignment of electronic dipole moments in the direction of the magnetic field that is used to magnetize the sphere. The uniform magnetization leads to an internal magnetic field that is also uniform and an external magnetic field that is identical to the field of a point magnetic dipole (Ref. 13, Sec. III and references therein). The south pole of the sphere is defined as the point where the magnetic field enters the sphere and is perpendicular to it. The north pole is defined as the point where the magnetic field exits the sphere and is perpendicular to it.

A single uniformly magnetized sphere can exhibit complex motions as it responds to the forces and torques exerted by a fixed but otherwise identical sphere. We recently studied the motion of a free sphere that slides without friction along the surface of a fixed sphere, with orbital and spin degrees of freedom for the free 
sphere but no radial motion. These assumptions give periodic nonlinear states and infinite cascades of mixed-mode bifurcations. ${ }^{14}$ We have also studied the dynamics of two freely rotating uniformly magnetized spheres that are in frictionless contact with each other. ${ }^{1}$

In this paper, we study the conservative dynamics of a free sphere that makes contact with a fixed sphere only during frictionless elastic hard-sphere collisions, and we, therefore, include orbital, spin, and radial degrees of freedom for the free sphere. Compared with our previous study of periodic sliding states, adding the radial degree of freedom means that three degrees of freedom must be synchronized instead of two in order to find periodic solutions.

Our study of bouncing periodic modes shares some characteristics with a billiard ball in a uniform gravitational field that collides elastically with a wedge ${ }^{16}$ or parabola. ${ }^{17}$ In these studies, the ball is treated as a point particle with two translational degrees of freedom (horizontal and vertical), and the concavity of the wedge or parabola enables localized periodic modes. For the parabola, there is a symmetric periodic mode in which the ball bounces vertically from the center of the parabola.

In contrast, our free sphere has a finite radius, two translational degrees of freedom (radial and orbital), and one rotational degree of freedom (spin). This sphere is subject to the nonuniform magnetic field of the fixed sphere, which exerts both forces and torques on the free sphere. The attraction of the free sphere toward its stable equilibrium position, with its south pole in contact with the north pole of the fixed magnet, enables localized periodic modes. There is a symmetric spinless periodic mode in which the free sphere bounces radially from its equilibrium position, and periodic modes with synchronized spin and orbital motions bifurcate from this radial mode. Key to these modes is the phase relationship between spin and orbital motions, which is irrelevant in the gravitational billiard problem.

We locate the free sphere initially at its stable equilibrium position and we search for periodic states by varying the initial orbital, spin, and radial momenta. We characterize such states by the number $m$ of bounces per period, the number of angular oscillations $n$ per period, and the phase relationship $p$ between the orbital and spin motions. A family of states with the same descriptor $(m, n, p)$ is called a "mode."

We find closed-form periodic solutions for small-amplitude motion near the equilibrium position and use these solutions to inform the search for finite-amplitude periodic solutions. This search yields 1497 finite-amplitude modes, of which 1243 are distinct. The bulk of this paper is devoted to identifying and characterizing these modes.

Details of our fourth-order Runge-Kutta integration of the equations of motion, our treatment of hard-sphere collisions, and our code validation by comparison with various analytical limits are discussed elsewhere (Ref. 14, Figs. 6-8 and Figs. 11-14) (Ref. 18, Figs. 1 and 2), (Ref. 19, Figs. 4-7). Further validation is supplied by the precise agreement of the threshold energies of our 1497 modes with our independent prediction of these energies (Ref. 18, Figs. 8 and 9), and by the constancy of the energy during simulations (Ref. 18, Sec. I, penultimate paragraph).
We take advantage of our recent proof that simple dipolar interactions exactly describe the magnetic interactions between uniformly magnetized spheres. ${ }^{13}$ In Paper II, we explore scaling relationships for the energy and period of periodic modes discussed in this paper. ${ }^{18}$

\section{MATHEMATICAL DESCRIPTION}

We describe the $2 \mathrm{D}$ position of the free sphere using polar coordinates $(r, \theta)$ and describe its magnetic orientation by $\phi$ (Fig. 1). The corresponding radial momentum $p_{r}$, orbital angular momentum $p_{\theta}$, and spin angular momentum $p_{\phi}$ bring the state-space dimensionality to six, and energy conservation supplies an algebraic relationship between these six dynamical variables that reduces the effective dimensionality to five.

For a magnetic sphere of radius $a$ with uniform magnetization $\mathbf{M}$, the total magnetic dipole moment is

$$
\mathbf{m}_{1}=\frac{4}{3} \pi a^{2} \mathbf{M}
$$

Placing this dipole at the coordinate origin (Fig. 1) produces a pure dipole magnetic field given by

$$
\mathbf{B}(\mathbf{r})=\frac{\mu_{0}}{4 \pi}\left(\frac{3 \mathbf{m}_{1} \cdot \mathbf{r}}{r^{5}} \mathbf{r}-\frac{\mathbf{m}_{1}}{r^{3}}\right)
$$

where $\mathbf{B}(\mathbf{r})$ is the magnetic field of the fixed sphere at the position $\mathbf{r}$ of the free sphere, of magnitude $r>a$.

The potential energy, force, and torque on the free sphere are the same as those for a point dipole and are given, respectively, by ${ }^{13}$

$$
\begin{gathered}
U=-\mathbf{m}_{2} \cdot \mathbf{B}, \\
\mathbf{F}=-\nabla U, \\
\boldsymbol{\tau}=\mathbf{m}_{2} \times \mathbf{B} .
\end{gathered}
$$

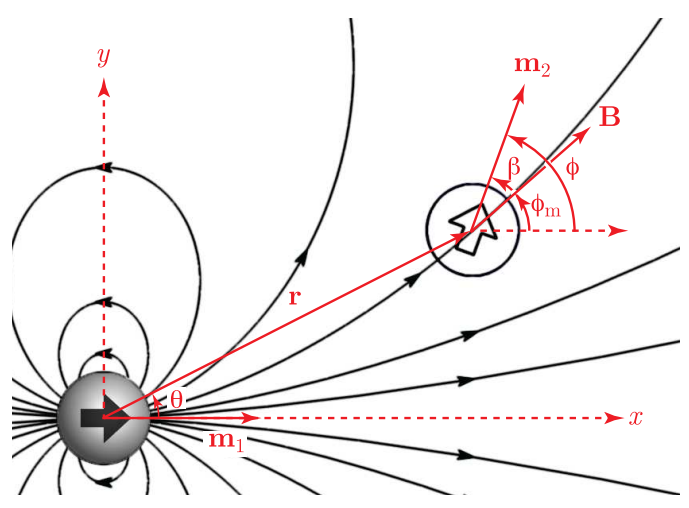

FIG. 1. Polar coordinates $(r, \theta)$ used to describe the $2 \mathrm{D}$ position of a free sphere with magnetic dipole moment $\mathrm{m}_{2}$, whose direction is given by the angle $\phi$, measured counterclockwise from the $+x$ direction. This sphere moves in response to the forces and torques produced by a sphere that is held fixed at the origin, with its magnetic moment $m_{1}$ fixed in the $+x$ direction and its magnetic field lines shown as directed traces. At the position $\mathbf{r}$ of the free sphere, $\phi_{m}$ gives the direction of the local magnetic field $\mathbf{B}$ and $\beta$ gives the direction of $\mathbf{m}_{2}$ relative to $\mathbf{B}$. 


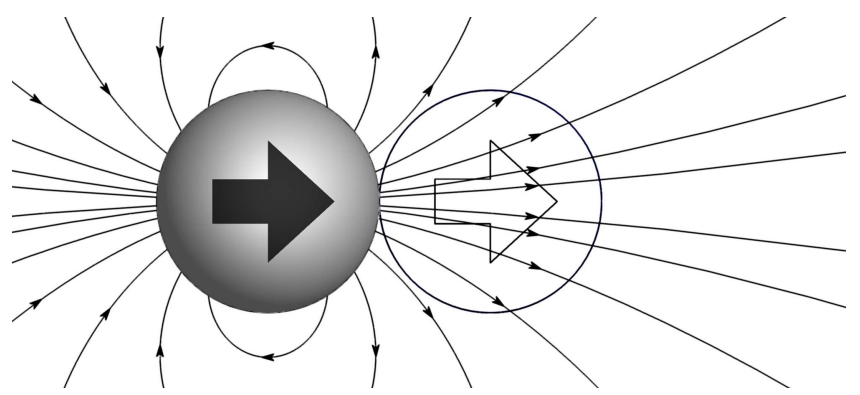

(a)

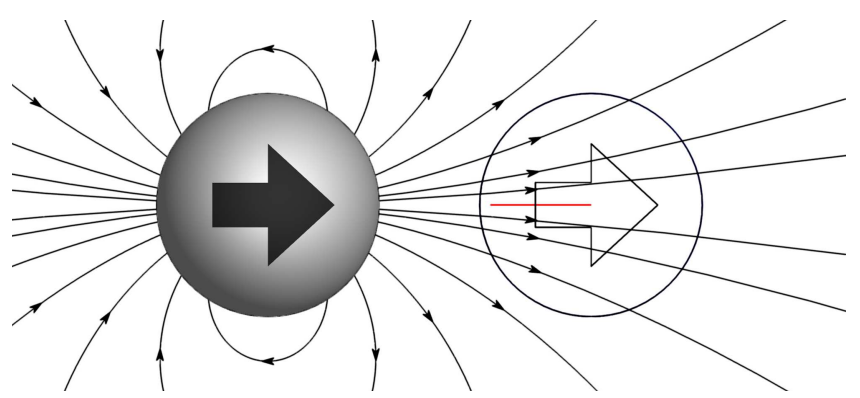

(b)

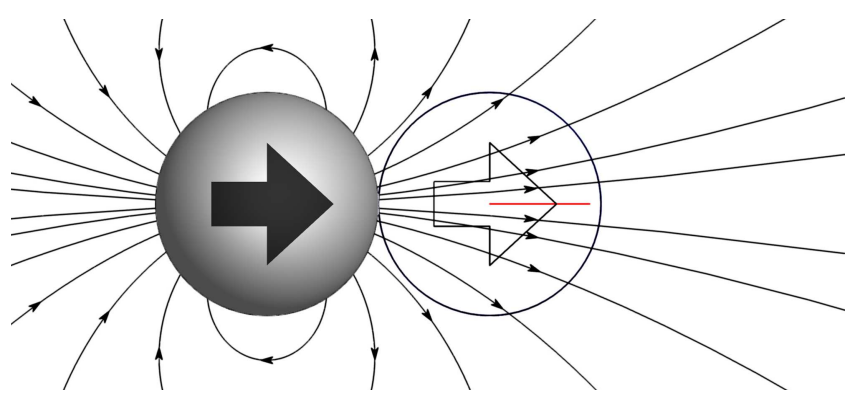

(c)

FIG. 2. MagPhyx snapshots of the radial bouncing mode discussed in Sec. III A, ${ }^{2}$ at times $t=0(\mathrm{a}), T / 2(\mathrm{~b})$, and $T(\mathrm{c})$, where $T$ is the period of the motion. The shaded sphere is fixed in position and orientation, and the outlined sphere bounces periodically on the fixed sphere. Arrows superposed over the spheres give the directions of their magnetic moments. Magnetic field lines are shown as directed traces, and the path of the motion of the free sphere is shown as a red trace.

The potential energy $U$ is lowest where $\mathbf{B}$ is strong and where $\mathbf{m}_{2}$ is aligned with $\mathbf{B}$. The force $\mathbf{F}$ on the free sphere is directed toward such regions, assuming that $\mathbf{m}_{2}$ is fixed in direction, and the torque $\boldsymbol{\tau}$ tends to rotate the free sphere in order to align $\mathbf{m}_{2}$ with $\mathbf{B}$.

The free and fixed spheres have identical radii $a$, identical dipole moment magnitudes $\left|\mathbf{m}_{1}\right|=\left|\mathbf{m}_{2}\right|=m_{\mathrm{d}}$, identical masses $\tilde{m}$, and identical moments of inertia $I=2 \tilde{m} a^{2} / 5$ (Fig. 1). The fixed sphere is placed at the origin and has fixed magnetic moment $\mathbf{m}_{1}=m_{\mathrm{d}} \hat{\mathbf{x}}$. The free sphere is located at

$$
\mathbf{r}=r \cos \theta \hat{\mathbf{x}}+r \sin \theta \hat{\mathbf{y}},
$$

and its dipole moment is given by

$$
\mathbf{m}_{2}=m_{\mathrm{d}} \cos \phi \hat{\mathbf{x}}+m_{\mathrm{d}} \sin \phi \hat{\mathbf{y}} .
$$

This dipole moment is oriented at an angle $\phi$ measured counterclockwise from the $+x$ direction (Fig. 1).

Equation (2) supplies the Cartesian components of the magnetic field at the center of the free sphere, ${ }^{14}$

$$
\mathbf{B}(\mathbf{r})=\frac{\mu_{0} m_{\mathrm{d}}}{8 \pi r^{3}}[(1+3 \cos 2 \theta) \hat{\mathbf{x}}+3 \sin 2 \theta \hat{\mathbf{y}}] .
$$

The direction of this field is given by

$$
\phi_{m}(\theta)=\tan ^{-1}\left(\frac{3 \sin 2 \theta}{1+3 \cos 2 \theta}\right),
$$

measured counterclockwise from the $+x$ direction. The magnetic displacement angle $\beta$ is defined as the angle between $\mathbf{m}_{2}$ and $\mathbf{B}(\mathbf{r})$ (Fig. 1),

$$
\beta=\phi-\phi_{m}(\theta) .
$$

If $\mathbf{m}_{2}$ points in the direction of $\mathbf{B}$, then $\beta=0$ and $\boldsymbol{\tau}=0$. If $\mathbf{m}_{2}$ and $\mathbf{B}$ are misaligned, then $\beta \neq 0$ and the torque $\boldsymbol{\tau}$ attempts to align $\mathbf{m}_{2}$ with $\mathbf{B}$.

Using dimensionless variables, the Hamiltonian and dynamical equations become ${ }^{14}$

$$
\begin{gathered}
E=\frac{p_{r}^{2}}{2}+\frac{p_{\theta}^{2}}{2 r^{2}}+5 p_{\phi}^{2}-\frac{1}{12 r^{3}}[\cos \phi+3 \cos (\phi-2 \theta)], \\
\dot{r}=p_{r}, \\
\dot{\theta}=\frac{p_{\theta}}{r^{2}}, \\
\dot{\phi}=10 p_{\phi}, \\
\dot{p}_{r}=\frac{p_{\theta}^{2}}{r^{3}}-\frac{1}{4 r^{4}}[\cos \phi+3 \cos (\phi-2 \theta)]+F_{\mathrm{N}}, \\
\dot{p}_{\theta}=\frac{1}{2 r^{3}} \sin (\phi-2 \theta), \\
\dot{p}_{\phi}=-\frac{1}{12 r^{3}}[\sin \phi+3 \sin (\phi-2 \theta)],
\end{gathered}
$$

where an impulsive normal force $F_{\mathrm{N}}$ is exerted by the fixed sphere on the free sphere when they are in instantaneous frictionless contact during elastic hard-sphere collisions. Two conditions must be met during such collisions: (a) the angle of incidence equals the angle of reflection and (b) the kinetic energy (both rotational and translational) is conserved (Ref. 19, Sec. 7.2) (Sec. IV). To find bouncing modes, we do not actually calculate $F_{\mathrm{N}}$, and find it simpler to satisfy these two conditions instead. When the spheres are not in contact, $F_{\mathrm{N}}=0$.

As is readily seen from these equations, the free sphere finds a stable equilibrium position at $(r, \theta, \phi)=(1,0,0)$, with $p_{r}=p_{\theta}=p_{\phi}=0$ and $E=-1 / 3$. This corresponds to orienting the magnetic moments of the two spheres along the line joining their 


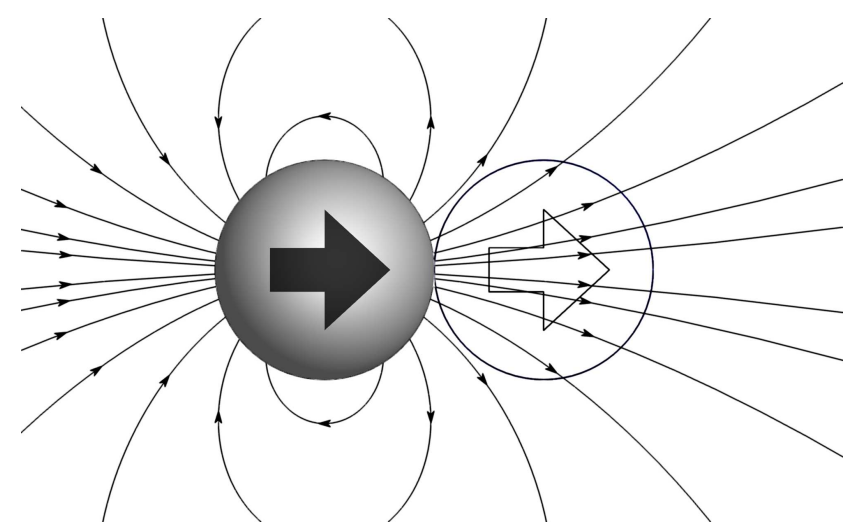

(a)

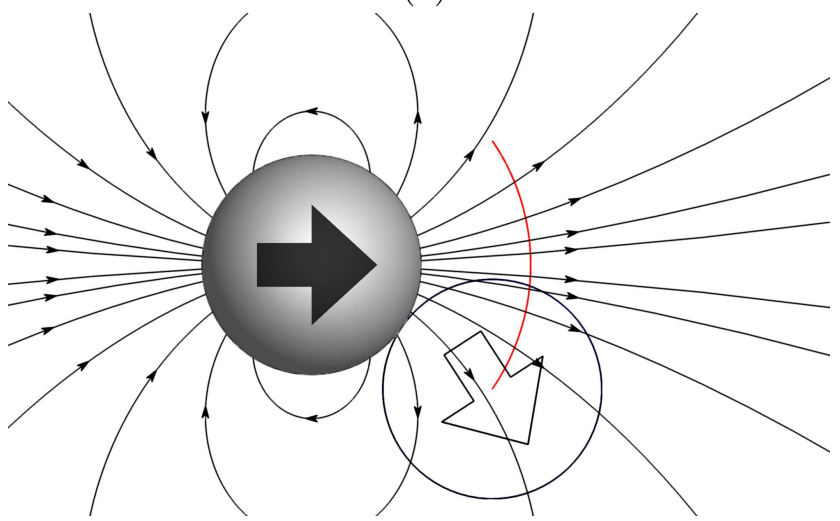

(c)

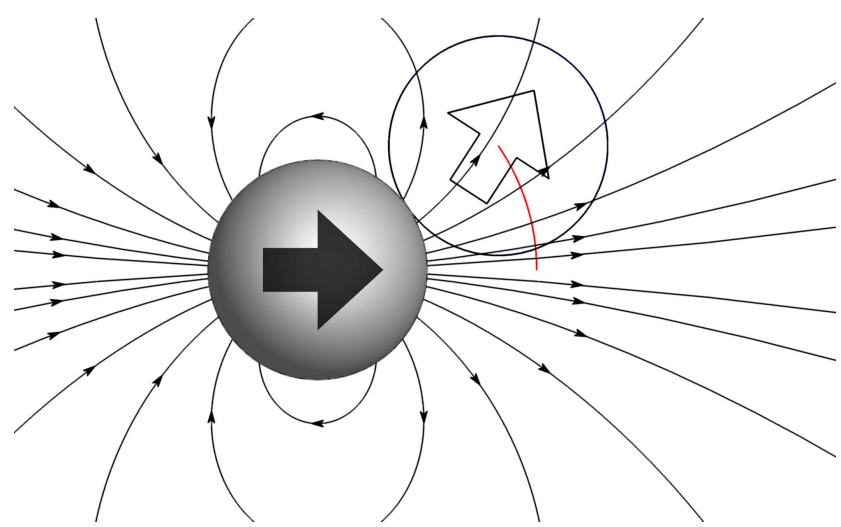

(b)

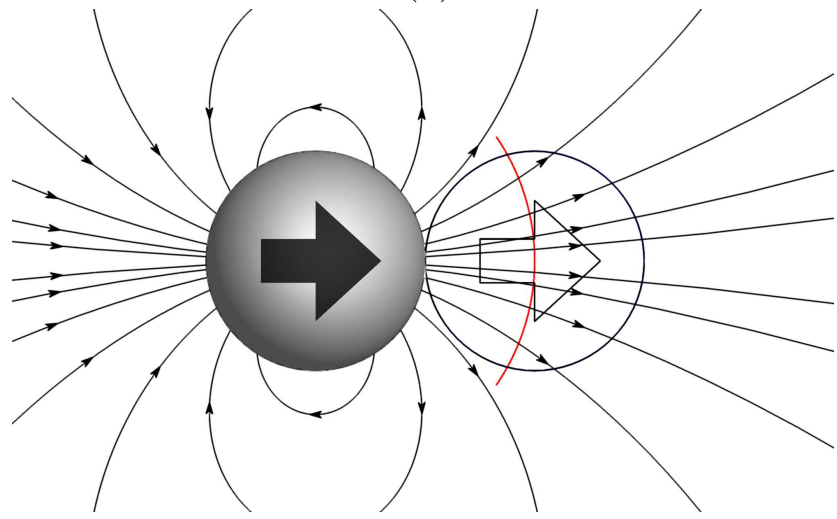

(d)

FIG. 3. MagPhyx snapshots of the in-phase angular sliding mode discussed in Sec. III B (mode 1), at times $t=0$ (a), $T / 4$ (b), $3 T / 4$ (c), and $T$ (d). In this mode, orbital motion dominates over spin motion.

centers, with the south pole of the free sphere in contact with the north pole of the fixed sphere. Below, we seek initial values of $p_{r}, p_{\theta}$, and $p_{\phi}$ that yield periodic states, with the free sphere located initially at the stable equilibrium position.

\section{SMALL-AMPLITUDE PERIODIC STATES}

In this section, we find small-amplitude periodic states in the vicinity of the stable equilibrium position, with synchronized radial, orbital, and spin motions. These states serve as a guide to our investigations of finite-amplitude periodic states and serve to validate our numerical results.

\section{A. Radial motion}

We first consider small-amplitude radial motion along the $x$ axis. We accordingly set $r(t)=1+\epsilon(t)$ and $\theta=\phi=p_{\theta}=p_{\phi}=0$ in Eqs. (11) to find

$$
E=-\frac{1}{3}+\epsilon+\frac{\dot{\epsilon}^{2}}{2}
$$

$$
\ddot{\epsilon}=-1 \text {. }
$$

These equations are valid when the spheres are not in contact $(\epsilon>0)$ and for small $\epsilon$. Thus, the free sphere experiences constant acceleration $\ddot{r}=\ddot{\epsilon}=-1$ directed toward the fixed sphere, like a particle moving under gravity near the earth's surface. We seek a relationship between the energy $E$ and the period $T_{r}$ of small-amplitude radial bouncing motions, with each bounce being a hard-sphere instantaneous elastic collision of the free sphere with the fixed sphere. To do so, we impose the initial conditions $\epsilon(0)=0$ and $\dot{\epsilon}(0)=v_{0}$, which apply immediately after the free sphere collides with the fixed sphere. In this way, the period $T_{r}$ is the time elapsed until the next collision. Integrating Eq. (12b) yields

$$
\epsilon(t)=v_{0} t-\frac{1}{2} t^{2}
$$

Substituting Eq. (13) into Eq. (12a) gives the conserved total energy,

$$
E=-\frac{1}{3}+\frac{v_{0}^{2}}{2}
$$




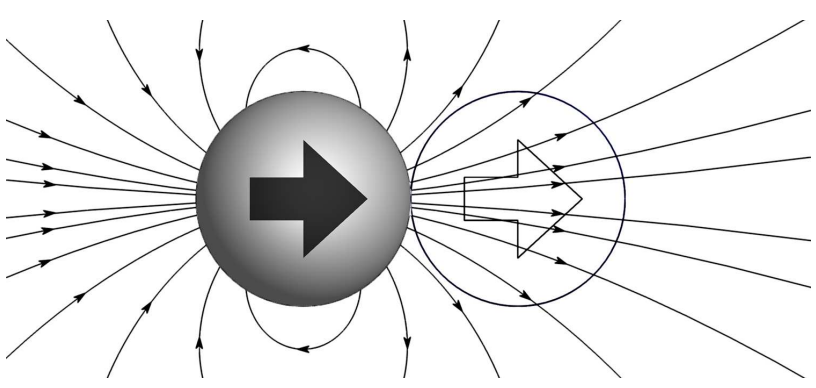

(a)

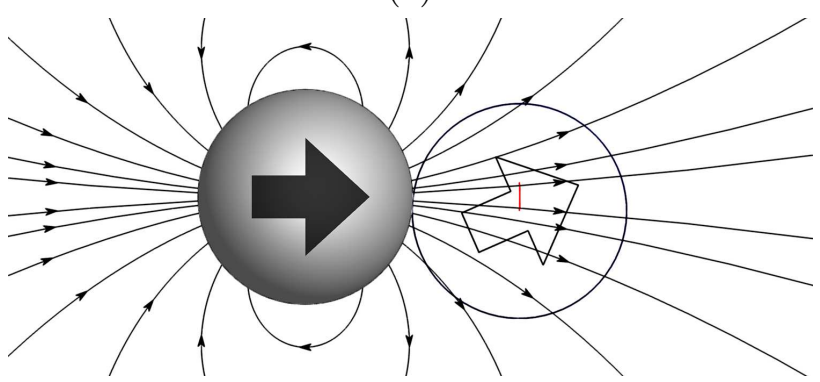

(c)

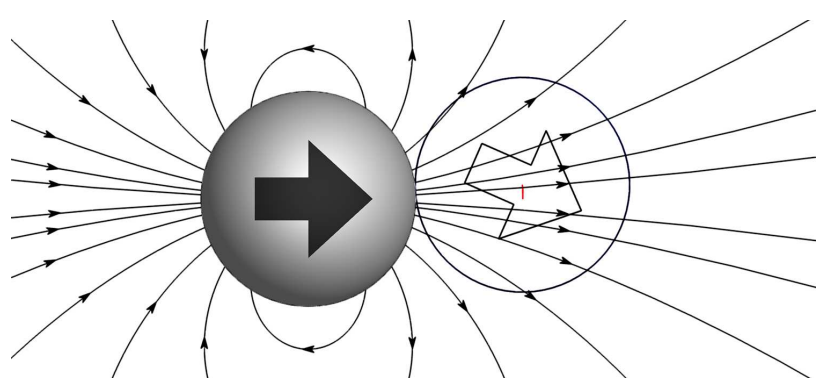

(b)

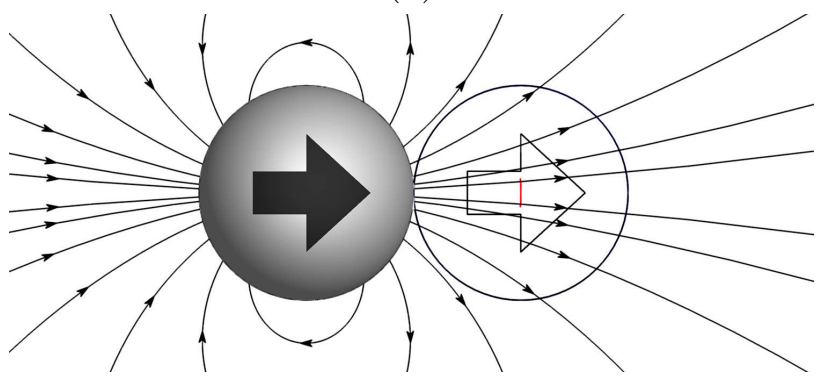

(d)

FIG. 4. MagPhyx snapshots of the out-of-phase angular sliding mode discussed in Sec. III B (mode 2), at times $t=0$ (a), $T / 4$ (b), $3 T / 4$ (c), and $T$ (d). In this mode, spin motion dominates over orbital motion.

Setting $\epsilon(t)=0$ in Eq. (13) at time $t=T_{r}$ gives the period of the bouncing motion,

$$
T_{r}=2 v_{0}
$$

Combining Eqs. (14) and (15) gives the desired relationship between the energy and the period,

$$
\begin{gathered}
E=-\frac{1}{3}+\frac{T_{r}^{2}}{8}, \\
T_{r}=\sqrt{8\left(E+\frac{1}{3}\right)} .
\end{gathered}
$$

MagPhyx free online software may be used to simulate this radial bouncing mode. ${ }^{20}$ Figure 2 shows MagPhyx snapshots of this mode at times $t=0, T_{r} / 2$, and $T_{r}$. MagPhyx Demo 1 visualizes the time-dependent motion of this mode. ${ }^{20}$ In the radial bouncing mode, the free sphere bounces periodically on the fixed sphere, with $\mathbf{m}_{2}$ always aligned with $\mathbf{B}$. Thus, the potential energy is associated with the movement of the sphere into regions of weaker magnetic field $\mathbf{B}$.

\section{B. Angular motion}

In this section, we describe small-amplitude spin and orbital oscillations of the free sphere about the stable equilibrium position, with no radial motion and with the free sphere maintaining frictionless contact with the fixed sphere $\left(r=1\right.$ and $\left.p_{r}=0\right)$. To first order in $\theta$ and $\phi$, Eq. (11) gives

$$
\left(\begin{array}{c}
\ddot{\theta} \\
\ddot{\phi}
\end{array}\right)=\left(\begin{array}{cc}
-1 & 1 / 2 \\
5 & -10 / 3
\end{array}\right)\left(\begin{array}{l}
\theta \\
\phi
\end{array}\right),
$$

and Eqs. (9) and (10) give

$$
\beta=\phi-\frac{3}{2} \theta
$$

We seek normal modes for which $\theta, \phi$, and $\beta$ oscillate at the same angular frequency $\omega$, of the form

$$
\begin{aligned}
& \theta(t)=C e^{i \omega t}, \\
& \phi(t)=D e^{i \omega t}, \\
& \beta(t)=E e^{i \omega t},
\end{aligned}
$$

where $C, D$, and $E$ are constants.

Equations (18) yield two normal modes. The lowest-frequency mode has an angular frequency $\omega_{1}$ given by

$$
\omega_{1}^{2}=\frac{13-\sqrt{139}}{6}
$$

period

$$
T_{1}=\frac{2 \pi}{\omega_{1}} \approx 14,
$$


TABLE I. Numbers $N_{1}$ and $N_{2}$ of in-phase and out-of-phase modes found for each bouncing number $m$ considered, and the numbers $n_{1}$ and $n_{2}$ of these modes that are unique, with values of $m$ that are prime numbers in bold typeface.

\begin{tabular}{lrrrr}
\hline \hline$m$ & $N_{1}$ & $N_{2}$ & $n_{1}$ & $n_{2}$ \\
\hline 1 & 0 & 20 & 0 & 20 \\
$\mathbf{2}$ & 0 & 17 & 0 & 9 \\
$\mathbf{3}$ & 1 & 13 & 1 & 10 \\
4 & 1 & 15 & 1 & 7 \\
$\mathbf{5}$ & 1 & 18 & 1 & 14 \\
6 & 1 & 20 & 1 & 5 \\
$\mathbf{7}$ & 3 & 21 & 3 & 19 \\
9 & 2 & 27 & 2 & 19 \\
$\mathbf{1 1}$ & 4 & 30 & 4 & 29 \\
$\mathbf{1 3}$ & 4 & 32 & 4 & 30 \\
15 & 3 & 35 & 3 & 21 \\
$\mathbf{1 9}$ & 5 & 42 & 5 & 41 \\
$\mathbf{2 3}$ & 5 & 49 & 5 & 49 \\
27 & 5 & 50 & 4 & 34 \\
$\mathbf{3 1}$ & 10 & 53 & 10 & 52 \\
39 & 10 & 64 & 6 & 40 \\
$\mathbf{4 7}$ & 11 & 70 & 11 & 70 \\
55 & 13 & 82 & 10 & 61 \\
63 & 7 & 94 & 4 & 56 \\
$\mathbf{7 9}$ & 13 & 93 & 13 & 93 \\
95 & 14 & 106 & 12 & 80 \\
11 & 8 & 128 & 5 & 82 \\
$\mathbf{1 2 7}$ & 12 & 121 & 12 & 121 \\
$\mathbf{1 5 7}$ & 11 & 153 & 11 & 153 \\
Totals & 144 & 1353 & 128 & 1115 \\
\hline \hline
\end{tabular}

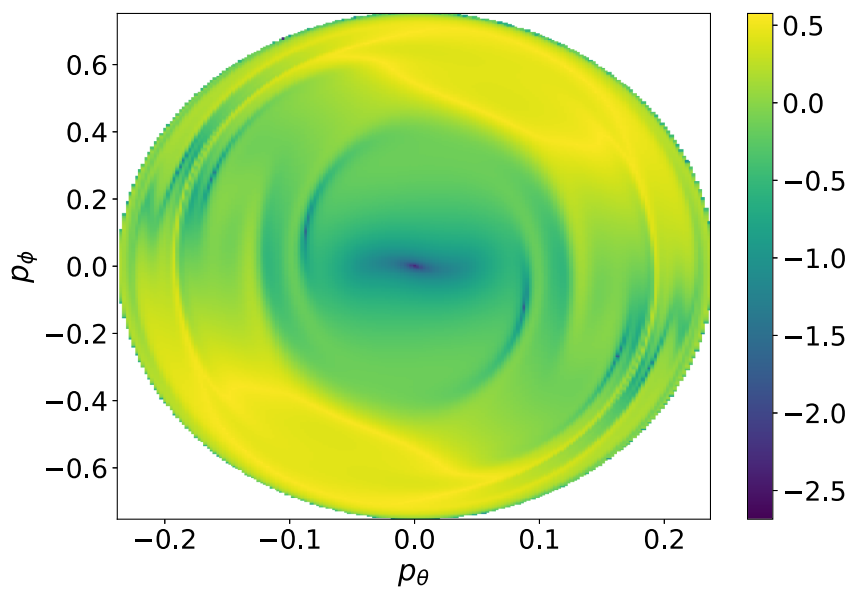

FIG. 5. Plot of $\log f\left[\mathbf{q}(0), t_{m}\right]$ as a function of $p_{\theta}(0)$ and $p_{\phi}(0)$, for $E=-0.05$ and $m=3$. The colorbar gives values of $\log f\left[q(0), t_{m}\right]$. The smallest value shown in the figure is about -2.5 , implying that there are no periodic solutions in this region, but this is an artifact of the discretization of the domain to display as an image. With infinitesimally small pixel size, multiple periodic solutions would be found.

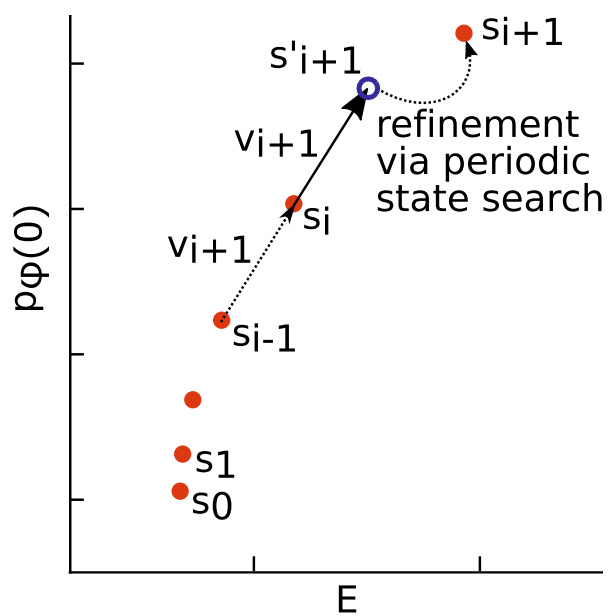

FIG. 6. Toy example showing our approach to tracing the states in a given mode. $p_{\theta}$ and $\mathbf{v}_{1}$ are omitted and distances between states are exaggerated for clarity. See Fig. 12 for states with $m=1$.

and phase relationships

$$
\begin{aligned}
\phi_{1} & =2\left(1-\omega_{1}^{2}\right) \theta_{1} \\
& \approx 1.6 \theta_{1},
\end{aligned}
$$

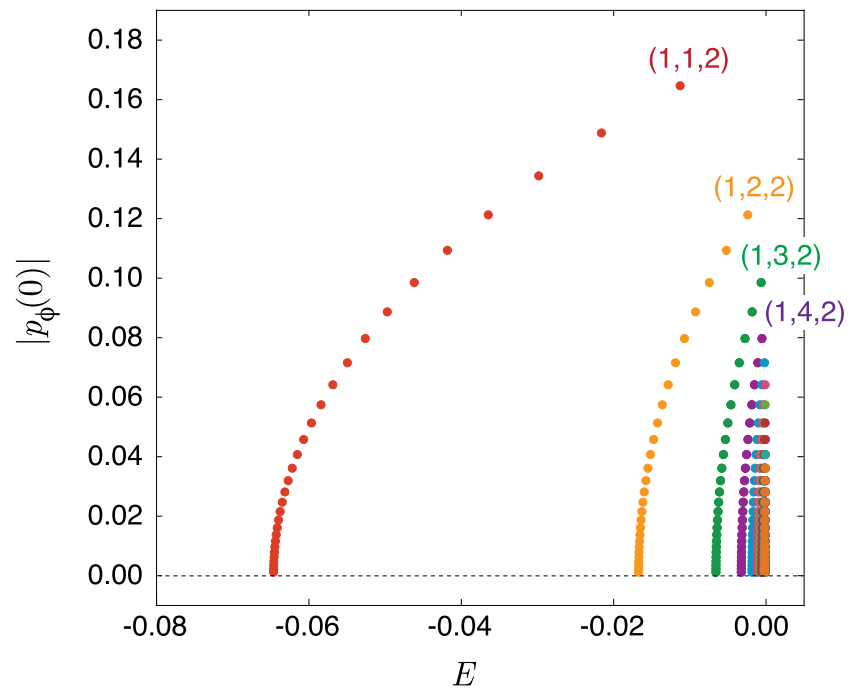

FIG. 7. Initial spin momentum $\left|p_{\phi}(0)\right|$ vs energy $E$ for finite-amplitude out-of-phase periodic states ( $p=2$, filled circles) with bouncing number $m=1$ and rocking numbers $n=1,2,3, \ldots$. Each mode $(m, n, p)$ has a family of states with different paired values of $E$ and $\left|p_{\phi}(0)\right|$, starting at a threshold energy $E=E_{m n p}$ with $\left|p_{\phi}(0)\right| \rightarrow 0$, and with $\left|p_{\phi}(0)\right|$ increasing with increasing $E>$ $E_{m n p}$. 
TABLE II. List of modes illustrated in this paper, together with demo numbers for associated MagPhyx visualizations. ${ }^{20}$

\begin{tabular}{|c|c|c|c|c|c|}
\hline Figure & Mode & $E$ & $T$ & $p_{\phi}(0)$ & Demo \\
\hline 2 & $(1,0,0)$ & -0.0988 & 3.98 & 0.0000 & 1 \\
\hline 3 & $(0,1,1)$ & -0.2900 & 14.76 & 0.0413 & 11 \\
\hline 4 & $(0,1,2)$ & -0.2900 & 3.15 & -0.0832 & 12 \\
\hline 8 & $(1,1,2)$ & -0.0112 & 5.85 & 0.1645 & 24 \\
\hline $9(\mathrm{a})$ & $(1,1,2)$ & -0.0646 & 6.16 & 0.0010 & 25 \\
\hline $9(b)$ & $(1,1,2)$ & -0.0596 & 6.14 & 0.0512 & 26 \\
\hline $9(\mathrm{c})$ & $(1,1,2)$ & -0.0461 & 6.09 & 0.0983 & 27 \\
\hline $9(d)$ & $(1,1,2)$ & -0.0112 & 5.85 & 0.1645 & 28 \\
\hline $10(a)$ & $(1,1,2)$ & -0.0461 & 6.09 & 0.0983 & 29 \\
\hline $10(b)$ & $(1,2,2)$ & -0.0074 & 20.60 & 0.0983 & 30 \\
\hline $10(\mathrm{c})$ & $(1,3,2)$ & -0.0006 & 45.07 & 0.0983 & 31 \\
\hline $11(\mathrm{a})$ & $(2,1,2)$ & -0.1306 & 4.31 & 0.0983 & 32 \\
\hline $11(\mathrm{~b})$ & $(2,3,2)$ & -0.0176 & 24.18 & 0.0983 & 33 \\
\hline $11(\mathrm{c})$ & $(2,5,2)$ & -0.0025 & 62.82 & 0.0983 & 34 \\
\hline 13(a) & $(3,1,2)$ & -0.0606 & 3.90 & 0.1819 & 35 \\
\hline 13(b) & $(3,2,2)$ & -0.0291 & 9.49 & 0.1819 & 36 \\
\hline $13(\mathrm{c})$ & $(3,4,2)$ & -0.0121 & 28.77 & 0.1211 & 37 \\
\hline $13(\mathrm{~d})$ & $(3,5,2)$ & -0.0118 & 43.29 & 0.1092 & 38 \\
\hline $13(\mathrm{e})$ & $(3,7,2)$ & -0.0049 & 82.66 & 0.0795 & 39 \\
\hline 13(f) & $(3,8,2)$ & -0.0058 & 107.38 & 0.0573 & 40 \\
\hline 14 & $(3,1,1)$ & -0.0068 & 123.08 & 0.0214 & 41 \\
\hline $15(\mathrm{a})$ & $(4,1,2)$ & -0.0861 & 3.64 & 0.1819 & 42 \\
\hline $15(\mathrm{~b})$ & $(4,3,2)$ & -0.0300 & 14.45 & 0.1645 & 43 \\
\hline $15(\mathrm{c})$ & $(4,5,2)$ & -0.0186 & 33.00 & 0.1211 & 44 \\
\hline $15(\mathrm{~d})$ & $(4,1,1)$ & -0.0186 & 64.14 & 0.0640 & 45 \\
\hline $15(\mathrm{e})$ & $(4,7,2)$ & -0.0067 & 60.86 & 0.0885 & 46 \\
\hline $15(\mathrm{f})$ & $(4,9,2)$ & -0.0082 & 101.92 & 0.0975 & 47 \\
\hline $16(\mathrm{a})$ & $(7,2,1)$ & -0.0141 & 161.36 & 0.0033 & 48 \\
\hline $16(\mathrm{~b})$ & $(7,2,1)$ & -0.0107 & 190.46 & 0.0456 & 49 \\
\hline $16(c)$ & $(11,2,1)$ & -0.0633 & 67.72 & 0.0245 & 50 \\
\hline $16(d)$ & $(11,2,1)$ & -0.0391 & 87.81 & 0.0885 & 51 \\
\hline $18(\mathrm{a})$ & $(79,1,1)$ & -0.1864 & 20.06 & 0.0714 & 52 \\
\hline $18(\mathrm{~b})$ & $(79,4,1)$ & -0.2218 & 67.24 & 0.0456 & 53 \\
\hline $18(\mathrm{c})$ & $(79,13,1)$ & -0.0663 & 414.73 & 0.0280 & 54 \\
\hline $18(d)$ & $(79,24,1)$ & -0.0109 & 2350.80 & 0.0159 & 55 \\
\hline 19 (a) & $(79,33,2)$ & -0.1650 & 126.45 & 0.0795 & 56 \\
\hline 19(b) & $(79,49,2)$ & -0.1046 & 218.23 & 0.1092 & 57 \\
\hline $19(\mathrm{c})$ & $(79,55,2)$ & -0.0859 & 257.55 & 0.1092 & 58 \\
\hline $19(\mathrm{~d})$ & $(79,57,2)$ & -0.0877 & 270.84 & 0.0983 & 59 \\
\hline $19(\mathrm{e})$ & $(79,63,2)$ & -0.0744 & 315.45 & 0.0885 & 60 \\
\hline $19(\mathrm{f})$ & $(79,74,2)$ & -0.0659 & 412.86 & 0.0512 & 61 \\
\hline 21(a) & $(79,67,2)$ & -0.0693 & 336.66 & 0.0456 & 62 \\
\hline 21(b) & $(79,94,2)$ & -0.0415 & 564.40 & 0.0245 & 63 \\
\hline
\end{tabular}

and

$$
\begin{aligned}
\beta_{1} & =\left(\frac{1}{2}-2 \omega_{1}^{2}\right) \theta_{1} \\
& \approx 0.097 \theta_{1} .
\end{aligned}
$$

Because $\theta_{1}$ and $\phi_{1}$ have the same sign, the orbital and spin oscillations are in phase. Because $\left|\beta_{1}\right| \ll\left|\theta_{1}\right|$, the magnetic moment $\mathbf{m}_{2}$ stays closely aligned with the local magnetic field $\mathbf{B}$ as the free sphere slides back and forth against the fixed sphere. ${ }^{14}$ Thus, the potential energy is dominated by the orbital movement of the sphere into regions of weaker magnetic field $\mathbf{B}$. Figure 3 illustrates this mode at a finite amplitude, and MagPhyx, Demo 11 visualizes it. ${ }^{20}$

The other mode has larger angular frequency $\omega_{2}$, given by

$$
\omega_{2}^{2}=\frac{13+\sqrt{139}}{6}
$$

smaller period

$$
T_{2}=\frac{2 \pi}{\omega_{2}} \approx 3.1,
$$

and phase relationships

$$
\begin{aligned}
\phi_{2} & =2\left(1-\omega_{2}^{2}\right) \theta_{2} \\
& \approx-6.3 \theta_{2},
\end{aligned}
$$

and

$$
\begin{aligned}
\beta_{2} & =\left(\frac{1}{2}-2 \omega_{2}^{2}\right) \theta_{2} \\
& \approx-7.8 \theta_{2} .
\end{aligned}
$$

Because $\theta_{2}$ and $\phi_{2}$ have opposite signs, the orbital and spin oscillations are $180^{\circ}$ out of phase. Because $\left|\beta_{2}\right| \gg\left|\theta_{2}\right|$, the direction of $\mathbf{m}_{2}$ departs significantly from the direction of $\mathbf{B}$, but the free sphere remains close to its equilibrium position as it slides against the fixed sphere. ${ }^{14}$ Thus, the potential energy is dominated by the spin misalignment of $\mathbf{m}_{2}$ with $\mathbf{B}$ rather than with the movement of the free sphere into regions of weaker magnetic field B. Figure 4 illustrates this mode at finite amplitude, and MagPhyx, Demo 12 visualizes it. ${ }^{20}$

\section{Radial and angular motion}

In this section, we describe periodic states with smallamplitude radial and angular oscillations about the stable equilibrium position. These states can be constructed by combining the small-amplitude motions discussed in Secs. III A and III B, noting that the period $T_{r}$ of the radial motion [Eq. (17)] depends on energy, with $T_{r} \rightarrow 0$ as $E \rightarrow-1 / 3$. Since the periods $T_{1}$ and $T_{2}$ of the in-phase and out-of-phase angular motions [Eqs. (21) and (25)] do not depend on energy, we can take advantage of the energy dependence of $T_{r}$ to find values of the energy for which the radial motion synchronizes with the angular motion.

For orbital and spin oscillations that are in phase with each other, we can synchronize angular and radial oscillations by writing the period of the overall motion as $T=m T_{r}=n T_{1}$, with a bouncing number $m$ giving the number of bounces per period and a rocking number $n$ giving the number of angular oscillations per period. We designate these in-phase modes by $(m, n, 1)$.

For orbital and spin oscillations that are out of phase with each other, we can synchronize angular and radial oscillations by writing the period of the overall motion as $T=m T_{r}=n T_{2}$, with $m$ 


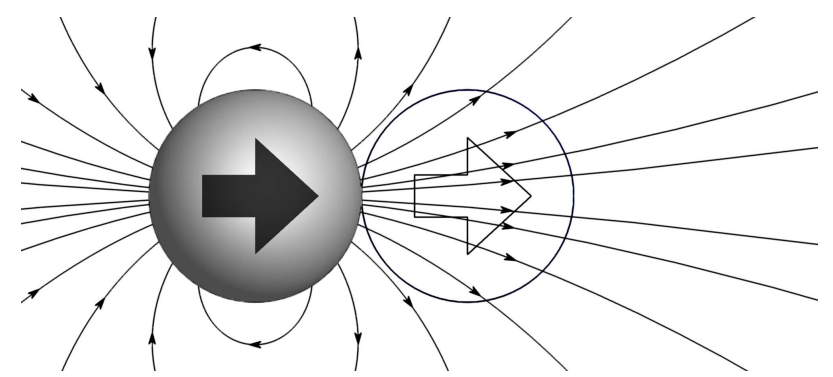

(a)

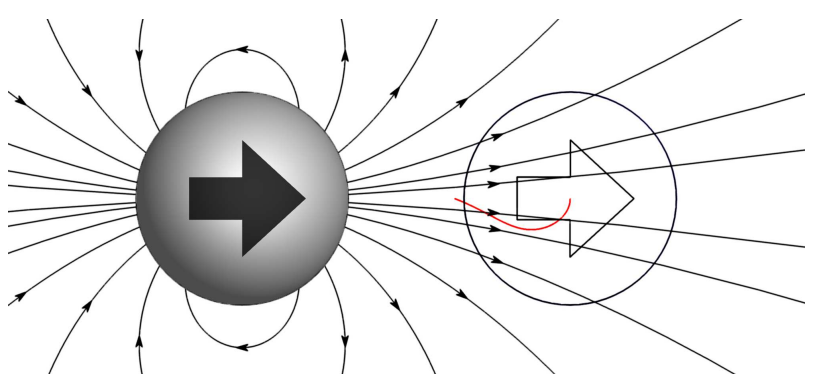

(c)

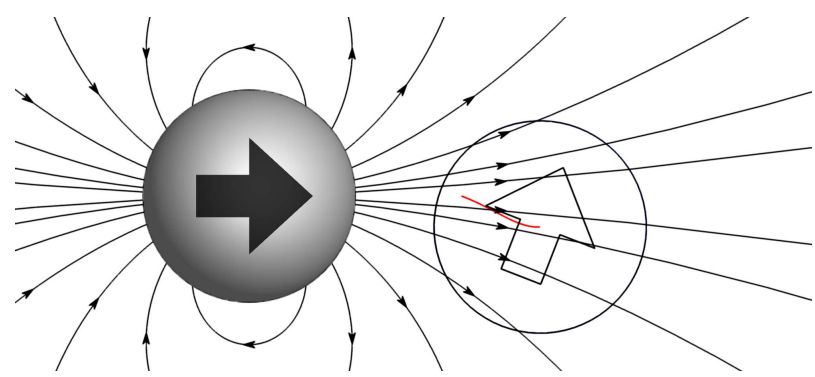

(b)

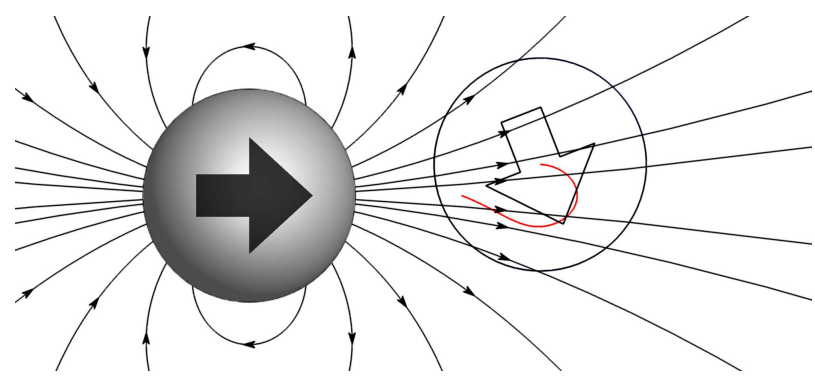

(d)

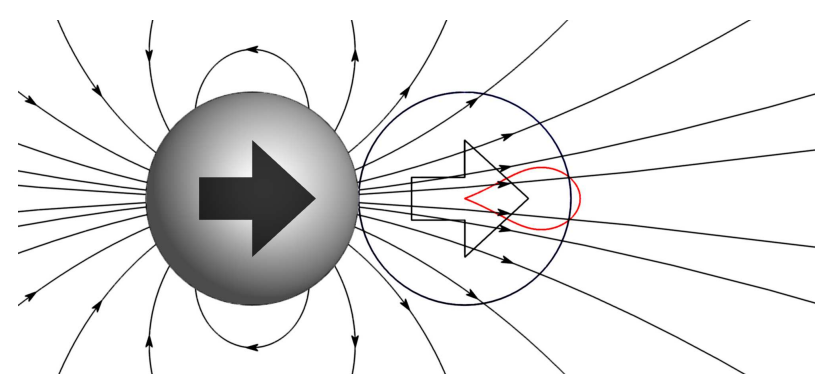

(e)

FIG. 8. MagPhyx snapshots of mode $(1,1,2)$ with $p_{\phi}(0)=0.1645$. In this mode, the spin and orbital motions are out of phase with each other, with spin motion dominating over orbital motion; the magnetic displacement angle covers a large range of $-78^{\circ}<\beta<+78^{\circ}$, while the orbital position angle is restricted to the narrow range of $-6.2^{\circ}<\theta<+6.2^{\circ}$.

and $n$ defined as before. We designate these out-of-phase modes by $(m, n, 2)$.

We designate modes generally by $(m, n, p)$, with $p=1$ for inphase modes and $p=2$ for out-of-phase modes. Writing the period as $T=m T_{r}=n T_{p}$ and applying Eq. (16) yields the small-amplitude energy and period, valid for $m \gg n$,

$$
\begin{gathered}
E_{m n p}=-\frac{1}{3}+\frac{n^{2} T_{p}^{2}}{8 m^{2}}, \\
T_{m n p}=n T_{p},
\end{gathered}
$$

where $T_{1}$ and $T_{2}$ are given by Eqs. (21) and (25), respectively.

We have just shown that small-amplitude periodic states have large bouncing numbers $m$ compared with their rocking numbers $n$. In these states, the bouncing frequency is high and the free sphere remains in close proximity to the fixed sphere.
Thus, there are two types of small-amplitude periodic states involving both radial and angular motion: one whose angular motion is dominated by orbital motion, with orbital and spin oscillations that are in phase $(p=1)$, and the other whose angular motion is dominated by spin motion, with orbital and spin oscillations that are out of phase $(p=2)$. The energies and periods of both types of states are given by Eqs. (28) and (29), with $m$ giving the number of bounces per period and $n$ giving the number of angular oscillations per period.

\section{FINITE-AMPLITUDE PERIODIC STATES}

We seek periodic motions of the free sphere with initial position $r(0)=1, \theta(0)=0, \phi(0)=0$ and initial nonzero momenta $p_{r}(0)>0, p_{\theta}(0)$, and $p_{\phi}(0)$. These initial conditions place the free sphere at the stable equilibrium position, with its magnetic moment 


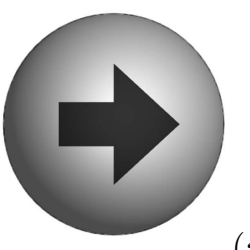

(a)

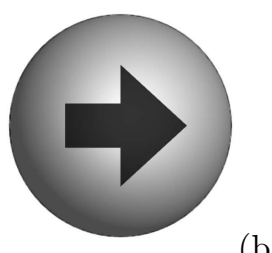

(b)

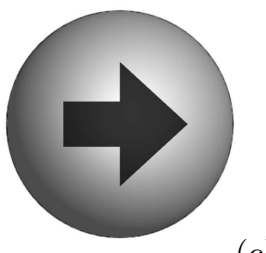

(c)

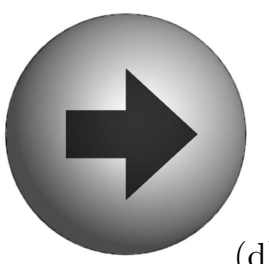

(d)

FIG. 9. MagPhyx trajectories for mode $(1,1,2)$ with $p_{\phi}(0)=0.0010$ (a), 0.0512 (b), 0.0983 (c), and 0.1645 (d). Frame (d) replicates the trajectory shown in Fig. 8.

aligned with the fixed sphere, and endow the sphere with nonzero radial momentum, orbital angular momentum, and spin angular momentum. Taking $p_{r}(0)>0$ means that, at the initial time, the free sphere has just completed a hard-sphere collision with the fixed sphere. Given that the initial position of the free sphere is always the same, it is the values of the initial momenta $p_{r}(0), p_{\theta}(0)$, and $p_{\phi}(0)$ that completely determine the subsequent motion of the sphere.

Because $E=-1 / 3$ is a minimum and because states with $E \geq 0$ are unbounded (meaning that the free sphere eventually drifts off to infinity), ${ }^{14}$ we seek periodic states with $-1 / 3<E<0$. Finding a particular mode $(m, n, p)$ is guaranteed only for $m \gg n$, for which Eqs. (28) and (29) pertain for small amplitudes.

Collisions occur when $r=1$ and $p_{r}<0$. During an elastic hard-sphere collision, the values of $p_{\theta}$ and $p_{\phi}$ remain the same, and $p_{r} \rightarrow-p_{r}$. This ensures that the angle of incidence equals the angle of reflection, and the kinetic energy is conserved during collisions. We consider the collisions to occur instantaneously.

Our initial conditions yield periodic states whose trajectories are symmetric under reflections about the $x$ axis, meaning that for every point $(r, \theta)$ on a periodic trajectory, there is a corresponding point $(r,-\theta)$ on that trajectory. This is easily seen by starting
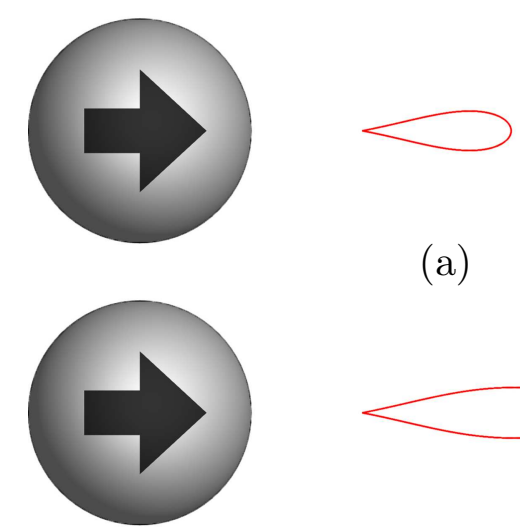

(a)
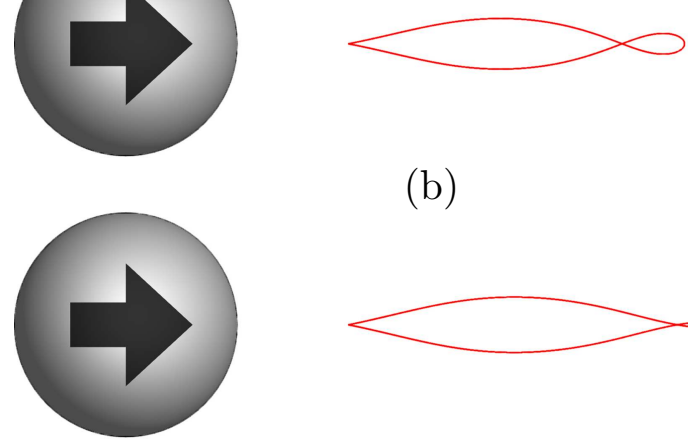

(b)

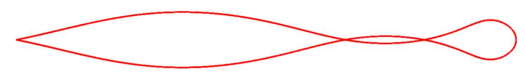

(c)

FIG. 10. Single-passage MagPhyx trajectories for modes $(1,1,2)(a),(1,2,2)(b)$, and $(1,3,2)(c)$, all with $p_{\phi}(0)=0.0093$. Frame (a) replicates the trajectory shown in Fig. $9(\mathrm{c})$.

from the same initial conditions as described above, but with time reversed, that is, $r(0)=1, \theta(0)=0, \phi(0)=0, p_{r}(0) \rightarrow-p_{r}(0)$, $p_{\theta}(0) \rightarrow-p_{\theta}(0)$, and $p_{\phi}(0) \rightarrow-p_{\phi}(0)$. Because $p_{r}(0)$ is now negative, the free sphere collides with the fixed sphere immediately after the free sphere is released, and this collision reverses the sign of $p_{r}(0)$ while leaving $p_{\theta}(0)$ and $p_{\phi}(0)$ the same. After this collision, we now have initial conditions that are symmetric about the $x$ axis relative to the original initial conditions. Thus, the symmetry of the initial position coupled with time reversal symmetry and hard-sphere collisions ensures that trajectories will be symmetric about the $x$ axis. We have not investigated initial conditions that yield non-symmetric states.

For a state to be periodic, the free sphere must return to its initial position and momentum, and the time required for this to happen is defined as the period $T$ of the motion. Once the initial momenta have been identified for a periodic state, we characterize the state using the same mode descriptors $(m, n, p)$ that we use to classify the small-amplitude periodic modes, with $p=1$ for in-phase modes and $p=2$ for out-of-phase modes, as follows:

If $p_{\theta}(0)$ and $p_{\phi}(0)$ have the same sign, then we set $p=1$ to reflect that the orbital and spin motions are initially in phase. To reflect the dominant role of orbital motion in this case, we define the rocking number $n$ as the number of times that the sign of $\theta$ changes from negative to positive during each period. We define the bouncing number $m$ as the number of hard-sphere collisions with the fixed sphere during each period, counting the final collision at time $t=T$ but not counting the initial contact at time $t=0$. For these conditions, the mode designation is $(m, n, 1)$. 


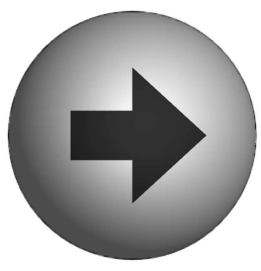

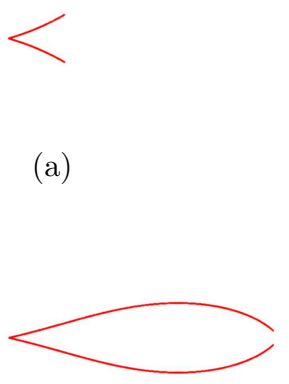

(b)
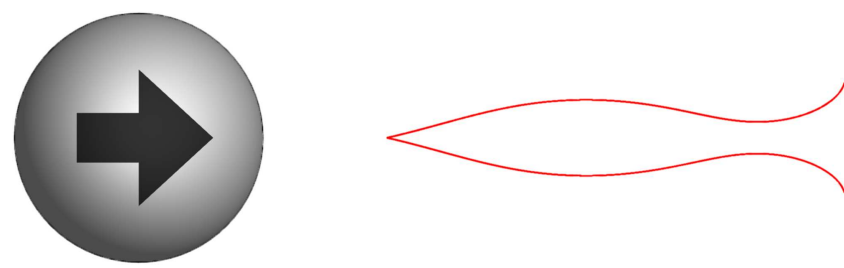

(c)

FIG. 11. Double-passage MagPhyx trajectories for modes $(2,1,2)(a),(2,3,2)$ (b), and $(2,5,2)(\mathrm{c})$, all with $p_{\phi}(0)=0.0093$.

If $p_{\theta}(0)$ and $p_{\phi}(0)$ have opposite signs, then we set $p=2$ to reflect that the orbital and spin motions are initially out of phase. To reflect the dominant role of spin motion in this case, we define the rocking number $n$ as the number of times that the sign of $\beta$ changes from negative to positive during each period. The angle $\beta$ offers a more reliable indicator of spin oscillations than $\phi$ because, when $\beta=0$, the magnetic moment of the free sphere points in the direction of the local magnetic field, so a sign change in $\beta$ means that the magnetic moment now points to the other side of the local magnetic field. The magnetic torque acts to rotate the magnetic moment back into alignment with the field. We define the bouncing number $m$ as before. For these conditions, the mode designation is $(m, n, 2)$.

If $m$ and $n$ have a common factor other than 1 , then mode $(m, n, p)$ is a duplicate of an irreducible mode $(m / k, n / k, p)$, where $k$ is the greatest common factor of $m$ and $n$. For example, for mode $(4,2,1), m=4$ and $n=2$ have a greatest common factor of 2 , and this mode is, therefore, a duplicate of the irreducible mode $(2,1,1)$. Indeed, the $(4,2,1)$ has the same energy as and twice the period of $(2,1,1)$ and simply executes the motion of $(2,1,1)$ twice. Irreducible modes $(m, n, p)$ are those for which $m$ and $n$ have no common factors other than 1 . To maximize the number of irreducible modes found in our finite-amplitude simulations, we give first preference to prime numbers and second preference to odd numbers in selecting values of $m$, while striving for even spacing of values of $m$ on a logarithmic scale.

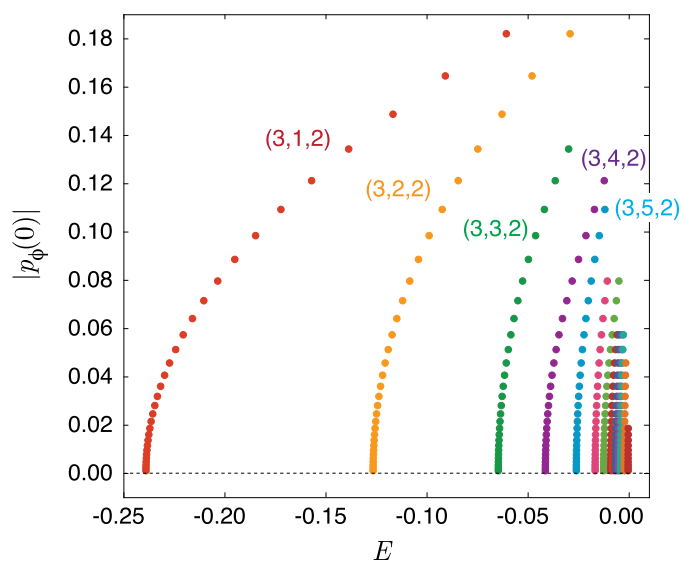

(a)

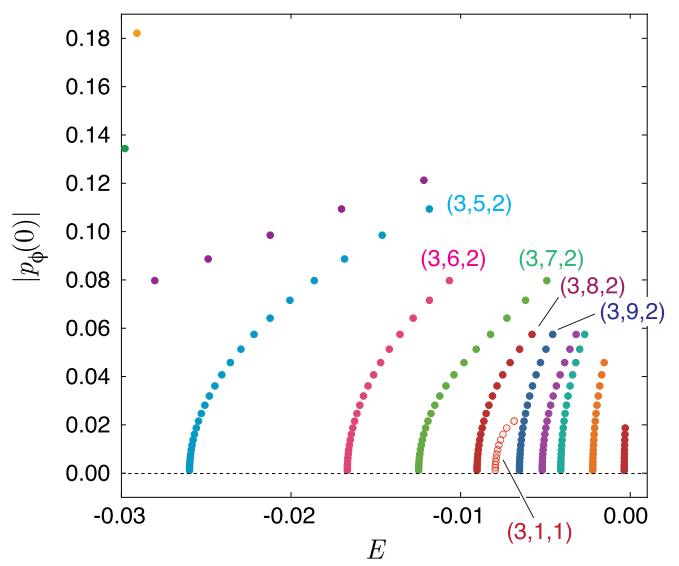

(b)

FIG. 12. Initial spin momentum $\left|p_{\phi}(0)\right|$ vs energy $E$ for finite-amplitude out-of-phase periodic states $(p=2$, filled circles) and in-phase periodic states ( $p=1$, open circles) with bouncing number $m=3$ and rocking numbers $n=1$, $2,3, \ldots$, with some mode families $(m, n, p)$ indicated. Frame (a) shows the entire range of energies and frame $(b)$ is a closeup of high-energy states.

Table I lists the values of $m$ considered, together with the number $N_{1}$ of in-phase ( $p=1$ ) modes and the number $N_{2}$ of outof-phase $(p=2)$ modes found for each bouncing number $m$, for a total of 144 in-phase modes and 1353 out-of-phase modes, and a grand total of 1497 modes found. Also listed in Table I are the number $n_{1}$ of irreducible in-phase modes and the number $n_{2}$ of irreducible out-of-phase modes for each bouncing number $m$, obtained by omitting duplicates, for a total of 128 irreducible in-phase modes and 1115 irreducible out-of-phase modes, and a grand total of 1243 irreducible modes found. Of these, there are two anomalous mode descriptors, $(7,2,1)$ and $(11,2,1)$, each of which associates with two distinct modes, each with its own energy, period, and motion (Sec. VI). Each of the other mode descriptors pertains to a single mode.

For example, we find only one mode $(4,2,1)$, and it is a duplicate of the irreducible mode $(2,1,1)$-we do not find another 
$(4,2,1)$ mode that is not a duplicate of mode $(2,1,1)$. Even though $(4,2,1)$ is a duplicate of another mode, it is the one-and-only $(4,2,1)$ mode, and, therefore, holds a place in the mode list for the purpose of analyzing trends in the mode energies and periods as functions of $m, n$, and $p$. For this reason, when performing such analyses below and in Ref. 18, we include both reducible and irreducible modes.

As discussed above, we define the rocking number $n$ using sign changes in $\theta$ for $p=1$ and using sign changes in $\beta$ for $p=2$. This definition is based on a selection process in which sign changes in $\theta$, $\phi, \beta, p_{\theta}$, and $p_{\phi}$ were considered as candidates. This definition minimizes the number of anomalous modes and ensures that all states in a family have the same mode descriptor $(m, n, p)$. With three different angles $(\theta, \phi$, and $\beta)$ and two angular momenta $\left(p_{\theta}\right.$ and $\left.p_{\phi}\right)$ to work with, it is remarkable that a single rocking number $n$, defined as above, suffices to associate each mode descriptor with a unique mode for all but two of our 1497 modes, these two being the two anomalous modes discussed above.

\section{NUMERICAL METHODS}

In this section, we describe our numerical methods for finding modes and periodic states within those modes. There are four specific methods we describe: magnet simulation, periodic state search, bifurcation search, and mode trace.

\section{A. Magnet simulation}

As described in detail in Ref. 19, we simulate a free magnet's interaction with a fixed magnet using fourth-order Runge-Kutta integration of Eqs. (11). For animation, the algorithm runs until stopped by the user, but when searching for periodic states, we restrict the algorithm to iterate until a specified number of bounces $m$ have occurred.

\section{B. Periodic state search}

As described in Sec. IV, a periodic state is one for which the free magnet returns to its initial position and momentum. The vector

$$
\begin{gathered}
\mathbf{q}(t)=\left[r(t), \theta(t), \phi(t), p_{r}(t), p_{\theta}(t), p_{\phi}(t)\right] \\
\equiv\left[q_{1}(t), q_{2}(t), q_{3}(t), q_{4}(t), q_{5}(t), q_{6}(t)\right]
\end{gathered}
$$

gives the six state variables $q_{i}(t)$ at time $t$, for $i=1,2, \ldots, 6$.

We consider the phase-space distance $f[\mathbf{q}(0), t]$ between states $\mathbf{q}(0)$ and $\mathbf{q}(t)$, where $\mathbf{q}(t)$ is obtained from the initial state $\mathbf{q}(0)$ by running the magnet simulation over a time $t$. The square of this distance is given by

$$
f^{2}[\mathbf{q}(0), t]=\sum_{i=1}^{6}\left[q_{i}(t)-q_{i}(0)\right]^{2} .
$$

Initially, we place the free sphere at its equilibrium position $(r, \theta, \phi)=(1,0,0)$, in contact with the fixed sphere so that

$$
\mathbf{q}(0)=\left[1,0,0, p_{r}(0), p_{\theta}(0), p_{\phi}(0)\right] .
$$

We run the magnet simulation until the free sphere makes its $m$ th collision with the fixed sphere and designate the time of this collision by $t=t_{m}$. We consider a state to be periodic if

$$
f\left[\mathbf{q}(0), t_{m}\right]<\varepsilon,
$$

where $\varepsilon=10^{-9}$. This criterion ensures that final values of the six state variables equal their initial values to within an error tolerance of $\varepsilon$, implying a periodic solution.

We use the Nelder-Mead Simplex algorithm ${ }^{21}$ to find minima of $f\left[\mathbf{q}(0), t_{m}\right]$. We search in $2 \mathrm{D}$ space, holding $p_{\phi}(0)$ constant and varying only $p_{\theta}(0)$ and $E$, where

$$
E=\frac{p_{r}^{2}(0)}{2}+\frac{p_{\theta}^{2}(0)}{2}+5 p_{\phi}^{2}(0)-\frac{1}{3}
$$

follows by inserting $\mathbf{q}(0)$ into Eq. (11a). If the minimum satisfies Eq. (34), it is considered to be periodic. A $2 \mathrm{D}$ projection of $f\left[\mathbf{q}(0), t_{m}\right]$ is shown in Fig. 5. We can see that the function is complicated with non-uniformly spaced minima, so finding minima through a $2 \mathrm{D}$ gridded search would require a large amount of computation even for modest resolution. Subsections $V \mathrm{C}-\mathrm{V}$ E describe our optimized search strategy.

How can the free sphere escape to infinity if it is initially in contact with the fixed sphere? The answer is that the free sphere is given an initial radial momentum, $p_{r}(0)$, orbital momentum $p_{\theta}(0)$, and spin momentum $p_{\phi}(0)$. If these values give $E>0$ from Eq. (35), the free sphere will eventually escape to infinity.

\section{Search for candidate bifurcations}

This and the following subsection describe efficient algorithms to find periodic states by finding bifurcations from the finiteamplitude radial mode and then tracing out the bifurcation in phase space.

To find potential bifurcations from the finite-amplitude radial mode for a given number of bounces $m$, we search at each point on a uniform grid of values $E(0) \in(-1 / 3,0)$ with spacing $10^{-3}$ and with $p_{\phi}(0)=10^{-3}$ and $p_{\theta}(0)=0$. We know that the periodic state search would not pull the state back to the finite-amplitude radial mode because the periodic state search varies only $p_{\theta}(0)$ and $E$, so $p_{\phi}(0)$ is guaranteed to remain at $10^{-3}$. At each grid point, if the periodic state search finds a periodic minimum within 1000 iterations, then we have found a candidate bifurcation with initial conditions $\mathbf{q}(0)$. In regions of particular interest, we also performed a number of custom searches with smaller grid spacing (step size).

In practice, we sometimes find candidate bifurcations that meet our periodicity criterion but are not true bifurcations. This especially occurs at low energy since the free magnet is moving very little. One approach to ensure that these states are not reported as true bifurcations would be to normalize $\varepsilon$ with a factor dependent on the total movement of the magnet, but this could quickly lead to floating point underflow. Instead, we wait until after tracing a mode (see the next step) before declaring a candidate bifurcation as a true bifurcation. 


\section{Mode trace}

Once we have found a candidate bifurcation, we trace out the mode in phase space (see Fig. 6). Given $\mathbf{q}(0)$, we define

$$
\mathbf{s}=\left[p_{\theta}(0), p_{\phi}(0), E\right],
$$

where $E$ is given by Eq. (35). We start our mode trace with the state $\mathbf{s}_{0}$ found by the bifurcation search [with $p_{\phi}(0)=0.001$ ] and seek subsequent states $\mathbf{s}_{1}, \mathbf{s}_{2}, \ldots, \mathbf{s}_{k}$ with increasing amounts of angular motion. To find $\mathbf{s}_{1}$, we do a periodic state search from $\mathbf{s}_{1}^{\prime}=\mathbf{s}_{0}+\mathbf{v}_{1}$, where

$$
\mathbf{v}_{1}=\left[p_{\theta, 0}, p_{\phi, 0}, 0\right]
$$

and where $p_{\theta, 0}$ and $p_{\phi, 0}$ are the $p_{\theta}(0)$ and $p_{\phi}(0)$ values for state $\mathbf{s}_{0}$, respectively. If the search starting at $\mathbf{s}_{1}^{\prime}$ finds a periodic state $\mathbf{s}_{1}$, we continue and look for $\mathbf{s}_{2}$. For $i \neq 0$, to find $\mathbf{s}_{i+1}$, we search from $\mathbf{s}_{i+1}^{\prime}=\mathbf{s}_{i}+\mathbf{v}_{i+1}$ where

$$
\mathbf{v}_{i+1}=\left[p_{\theta, i}-p_{\theta, i-1}, p_{\phi, i}-p_{\phi, i-1}, E_{i}-E_{i-1}\right],
$$

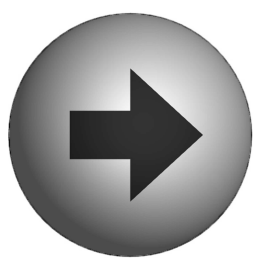<smiles>[C]1C2=CC1C2</smiles>

(a)
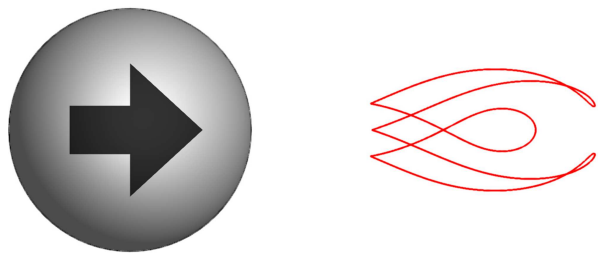

(c)

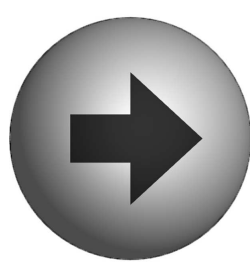

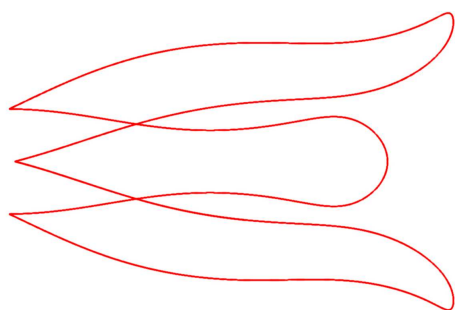

(e) and if the search yields a periodic minimum, we call it $\mathbf{s}_{i+1}$. This approach uses principles of both the Euler method and finite differences, where we use finite differences to approximate the derivative for the Euler step, but then we use the periodic state search to refine our Euler guess, moving it onto the true mode trace curve. The search ends when the periodic state search fails.

After tracing the states of the mode, we look at the last state found, $\mathbf{s}_{k}$. If $p_{\phi, k}>10^{-3}$, then we consider the mode to be legitimate. Otherwise, the candidate bifurcation is spurious and we discard it.

\section{E. Implementation}

We have two implementations of the magnet simulation algorithm. The first is in Javascript and is used in the MagPhyx software to visualize magnet interactions. This implementation provides not only an animation available on the MagPhyx website, but also the path traces shown in this paper (e.g., Fig. 19). The second implementation is done in $\mathrm{C}++$ for performance and is used in searching for periodic states.

The periodic state search is implemented in $\mathrm{C}++$ and uses the Gnu Scientific Library's ${ }^{22}$ implementation of the Simplex algorithm.
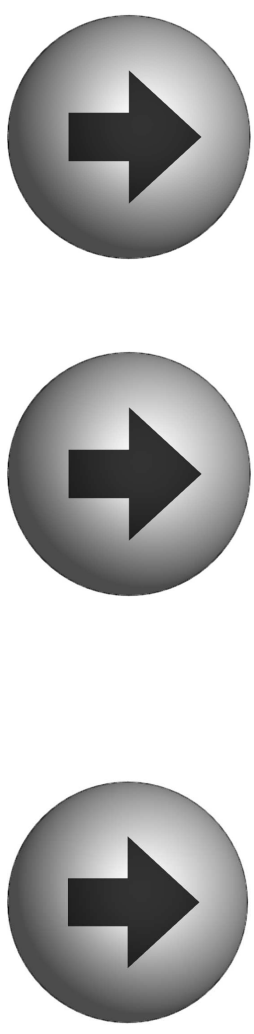

(f)

FIG. 13. Single-passage MagPhyx trajectories for irreducible modes $(3,1,2),(3,2,2),(3,4,2),(3,5,2),(3,7,2)$, and $(3,8,2)$. 


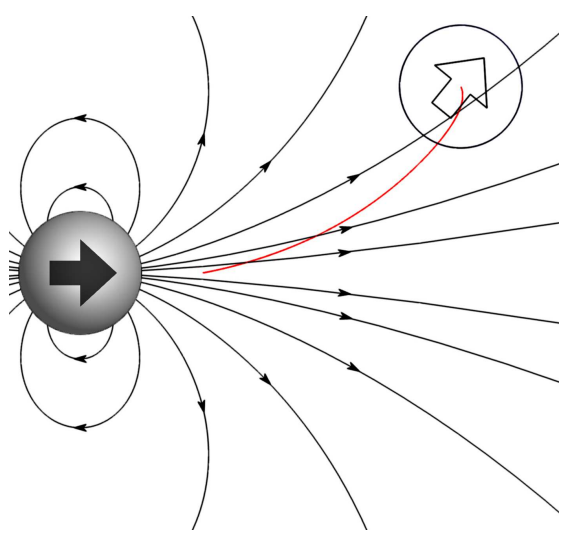

(a)

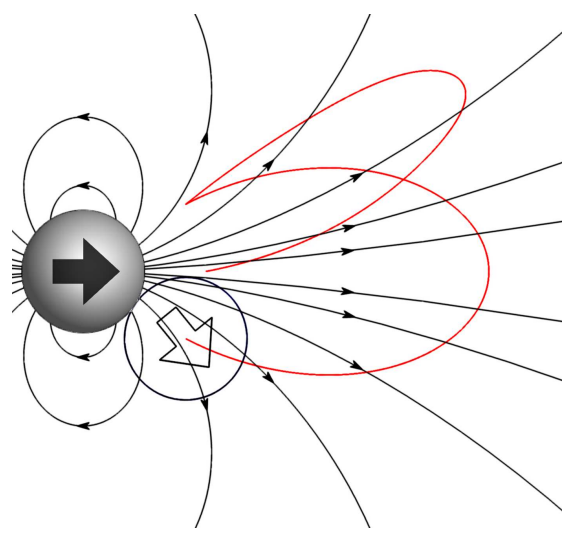

(d)

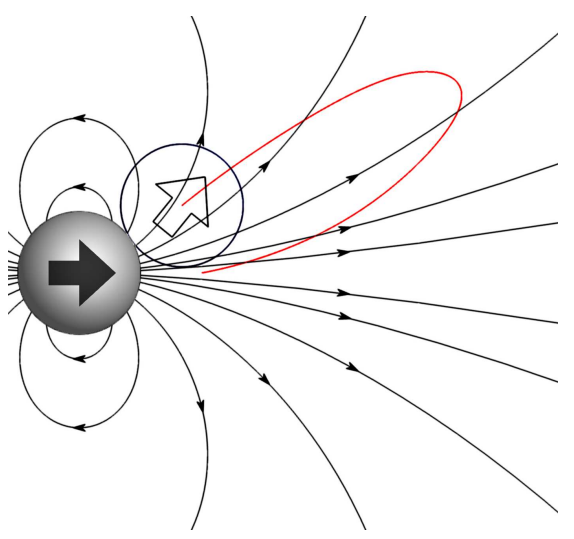

(b)

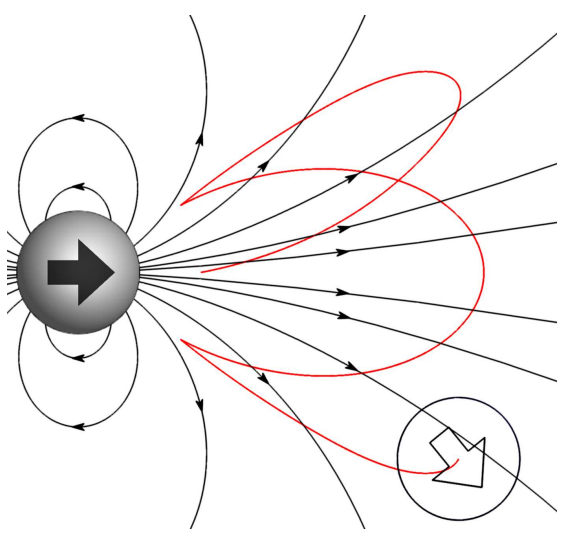

(e)

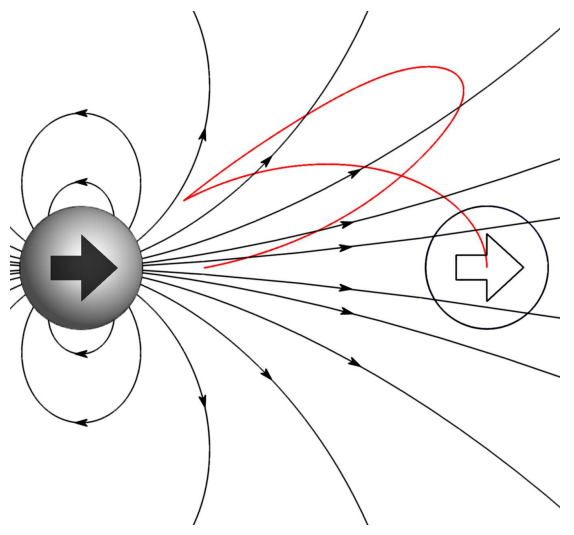

(c)

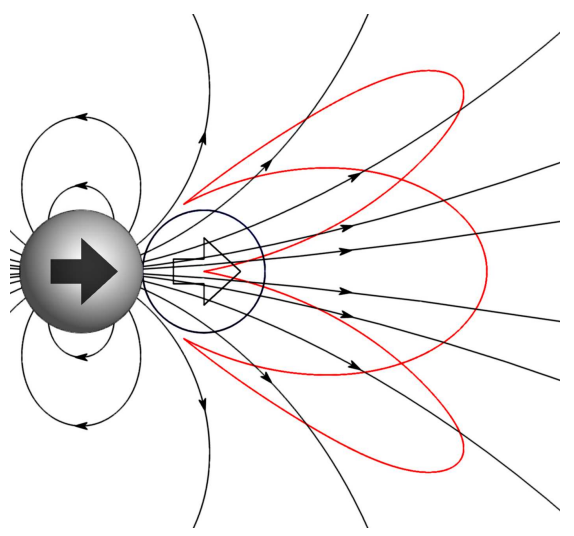

(f)

FIG. 14. MagPhyx snapshots of mode $(3,1,1)$ with $p_{\phi}(0)=0.0214$. In this mode, the spin and orbital motions are in phase with each other, with orbital motion dominating over spin motion; the orbital position angle covers a large range of $-35^{\circ}<\theta<+35^{\circ}$, while the magnetic displacement angle is restricted to $-11^{\circ}<\beta<+11^{\circ}$.

The search for candidate bifurcations and mode tracing is both done in Python, calling the $\mathrm{C}++$ subroutine to search for periodic states.

MagPhyx simulation software, ${ }^{20}$ MagPhyx code, ${ }^{23}$ data visualizations, ${ }^{24}$ and $\mathrm{C}++\operatorname{code}^{25}$ are all publicly available.

\section{TRAJECTORIES}

In this section, we describe the results of our findings for finiteamplitude states.

Figure 7 is a plot of $\left|p_{\phi}(0)\right|$ vs $E$ showing periodic out-of-phase states ( $p=2$, filled circles) with bouncing number $m=1$ and rocking numbers $n=1,2,3, \ldots$. Families of states are labeled by their mode descriptors $(m, n, p)$. States within each family differ in the amplitude of their angular motion but otherwise behave similarly. Each family has a continuum of states with a continuous range of values of $\left|p_{\phi}(0)\right|$ and $E$; only a discrete subset of this continuum is shown in Fig. 7. For $m=1$, only out-of-phase states $(p=2)$ are found, with $p_{\phi}(0)$ and $p_{\theta}(0)$ having opposite signs. For each state with positive initial spin momentum $p_{\phi}(0)$, there is a corresponding state with the same energy and negative initial spin momentum $-p_{\phi}(0)$.

Figure 7 illustrates how the finite-amplitude radial mode bifurcates into modes with angular motion. In the finite-amplitude radial mode, the magnetic moments of the two spheres point in the $+x$ direction and the free sphere travels along the $+x$ axis, with its south pole bouncing repeatedly against the north pole of the fixed sphere-there is no spin or orbital motion. As the energy increases, modes $(m, n, p)$ first appear with infinitesimal angular motion, $p_{\phi}(0) \rightarrow 0$ and $p_{\theta}(0) \rightarrow 0$. We designate this minimumenergy state as the threshold state, with energy $E_{m n p}$ and period $T_{m n p}$. As the energy increases beyond this threshold for mode $(m, n, p)$, the amplitude of the angular motion increases. Thus, the threshold energy $E_{m n p}$ and period $T_{m n p}$ correspond to finite-amplitude radial motion and infinitesimal-amplitude angular motion.

The lowest-energy mode in Fig. 7 is the $(1,1,2)$ mode. In this mode, the free sphere completes one bounce $(m=1)$ and one rocking cycle $(n=1)$ per period, with spin and orbital oscillations that are out of phase with each other $(p=2)$. Figure 8 


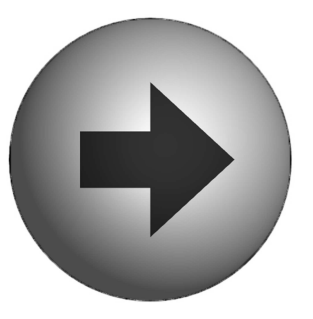

(a)
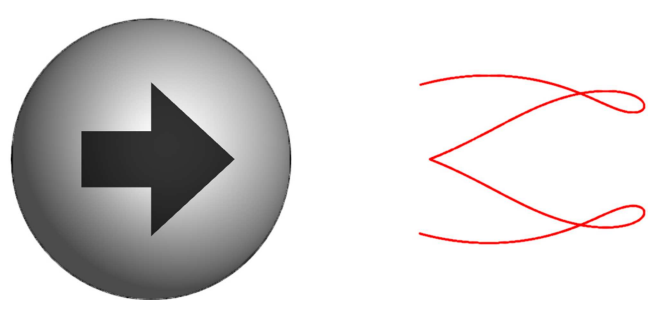

(c)
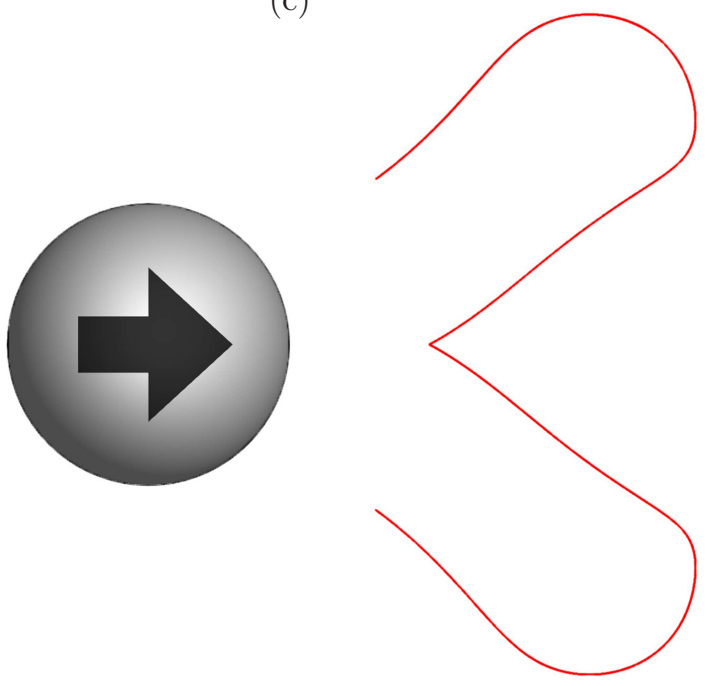

(e)
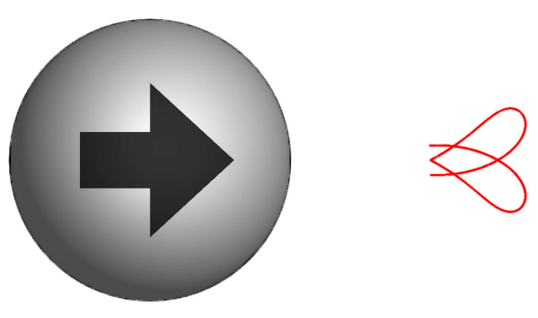

(b)

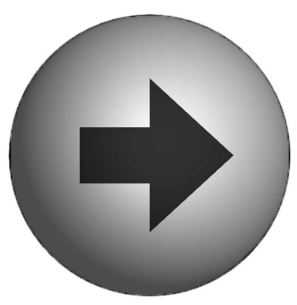

(d)
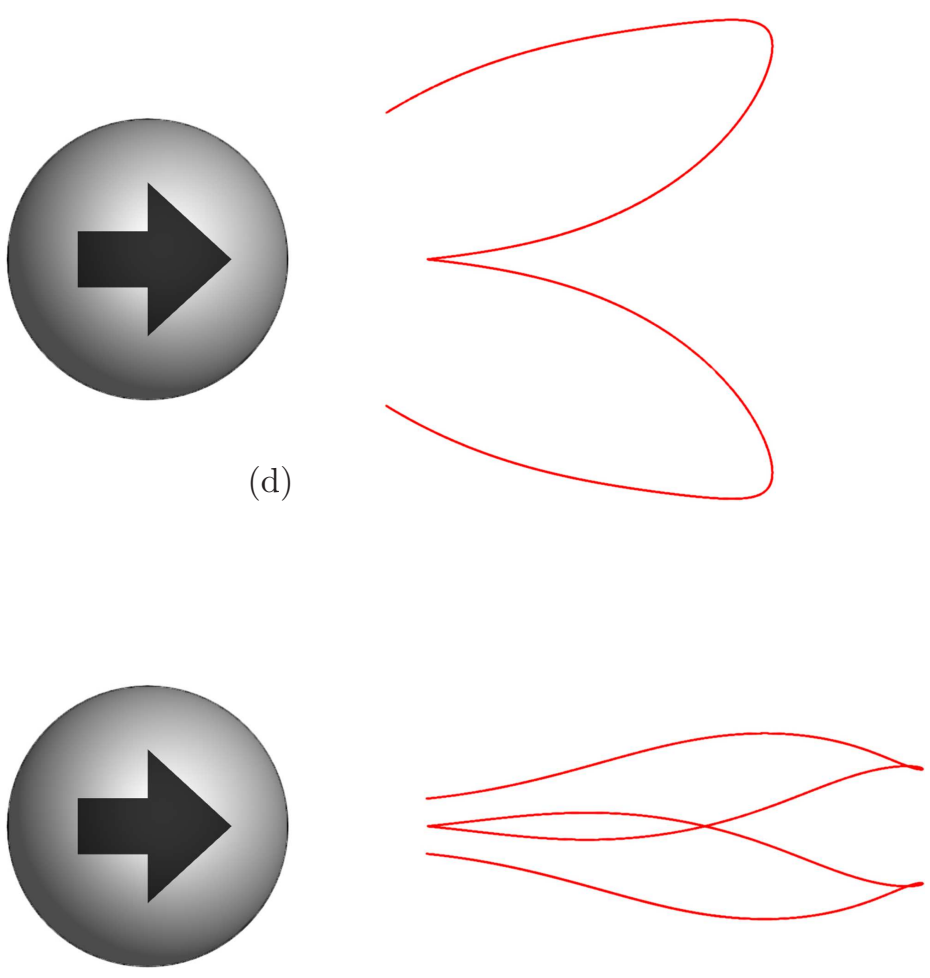

(f)

FIG. 15. Double-passage MagPhyx trajectories for irreducible modes (4,1,2), $(4,3,2),(4,5,2),(4,1,1),(4,7,2)$, and $(4,9,2)$.

shows a series of MagPhyx snapshots of the motion of the free sphere in this mode during one period of the motion, and Fig. 9 shows how the amplitude of the angular motion increases with increasing energy. Table II gives the corresponding values of $E$, $T$, and $p_{\phi}(0)$ and refers to online MagPhyx visualizations of these states.
The second-lowest energy mode in Fig. 7 is the $(1,2,2)$ mode, which exhibits two rocking cycles per period $(n=2)$ but is otherwise similar to the $(1,1,2)$ mode. The third-lowest energy mode is the $(1,3,2)$ mode, which exhibits three rocking cycles per period. Shown in Fig. 10 are MagPhyx trajectories for these three modes. Other one-bounce modes in Fig. 7 behave similarly, differing only in 

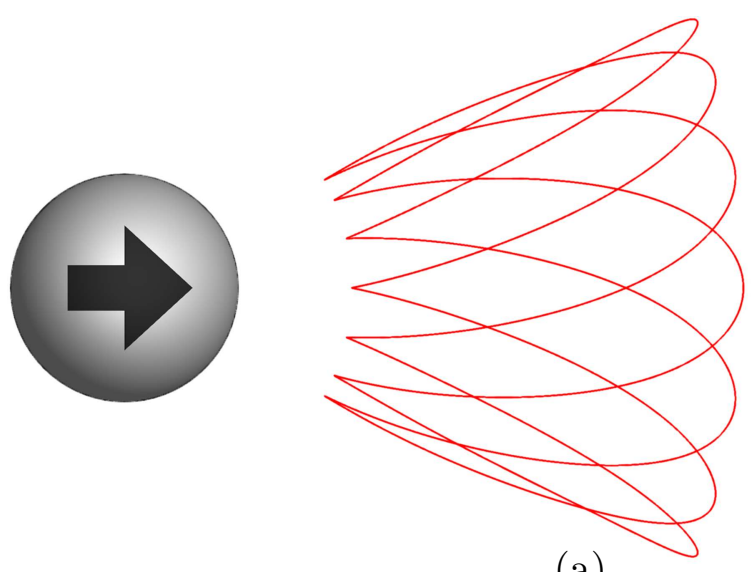

(a)

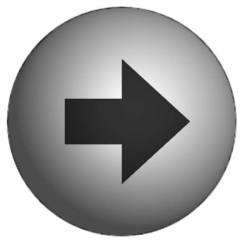

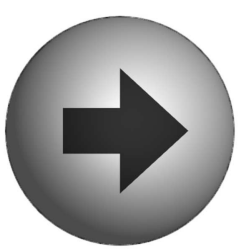

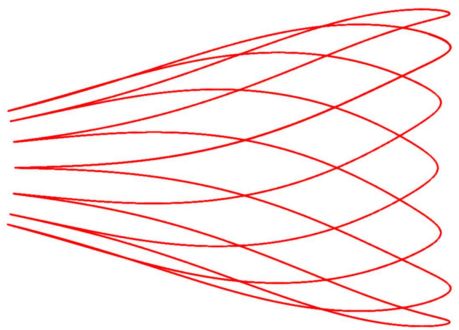

(b)

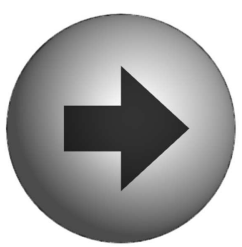

(c)

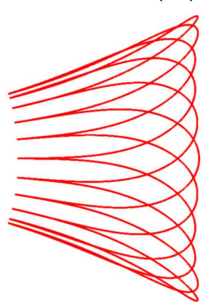

(d)

FIG. 16. Single-passage MagPhyx trajectories for anomalous irreducible modes $(7,2,1)(a)$ and (b) and $(11,2,1)(c)$ and (d).

the number of rocking cycles. All $m=1$ modes have single-passage trajectories, meaning that the free sphere passes along the trajectory just once per period.

For bouncing number $m=2$, the plot of $\left|p_{\phi}(0)\right|$ vs $E$ is similar to Fig. 7, except that it has more states and lower-energy states. The lowest of these is mode $(2,1,2)$, whose trajectory is shown in Fig. 11(a). In this mode, the free sphere bounces twice against the fixed sphere during each period of the motion, with both bounces occurring at the same place, $\theta=\phi=0$, and with $p_{\theta}>0$ for one bounce and $p_{\theta}<0$ for the other. The free sphere has a doublepassage trajectory, that is, it passes twice along the same path during each period of the motion, once in each direction.

It is also instructive to consider $m=2$ modes with $n>1$. Modes $(2,2,2)$ and $(2,4,2)$ are duplicates of modes $(1,1,2)$ and $(1,2,2)$ shown in Figs. 10 (a) and 10(b). Modes $(2,3,2)$ and $(2,5,2)$ are unique and are shown in Figs. 11(b) and 11(c). All unique $m=2$ modes have double-passage trajectories, whereas $m=1$ modes (and their $m=2$ duplicates) have single-passage trajectories.

For $m=2$ modes with $n=1,5,9, \ldots$, the two sides of the trajectory diverge at the end, like a vase, with the number of lobes in the trajectory increasing with increasing $n$ [Figs. 11(a) and 11(c)]. For $n=3,7,11, \ldots$, the two sides of the trajectory approach but do not touch each other at the end, like a pair of open tweezers [Fig. 11(b)].

Bouncing number $m=3$ is the smallest bouncing number that has in-phase states $(p=1)$. Figure 12 shows $\left|p_{\phi}(0)\right|$ vs $E$ for $m=3$, with out-of-phase states $(p=2)$ denoted by closed circles and in-phase states $(p=1)$ denoted by open circles. As $E$ increases, there are eight out-of-phase modes (for $n=1,2, \ldots, 8$ ) before the first in-phase mode $(3,1,1)$ is seen. Of these eight modes, $(3,3,2)$ is a duplicate of $(1,1,2)$ and $(3,6,2)$ is a duplicate of $(1,2,2)$. Singlepassage MagPhyx trajectories for the remaining six out-of-phase modes are shown in Fig. 13.

The first and only in-phase mode for $m=3$ is the $(3,1,1)$ mode, which executes one cycle of in-phase rocking motion while bouncing three times. Shown in Fig. 14 are snapshots of its singlepassage trajectory at different points during one period of its motion. Note that, in this mode, the free dipole is generally aligned closely with the magnetic field, and the oscillations in $\theta$ and $\phi$ are in phase.

For bouncing number $m=4$, the plot of $\left|p_{\phi}(0)\right|$ vs $E$ is similar to Fig. 12 and again features just one in-phase mode, $(4,1,1)$. Shown in Fig. 15 are MagPhyx double-passage trajectories for the first few irreducible $m=4$ modes.

Mentioned in Sec. IV are two anomalous mode descriptors, $(7,2,1)$ and $(11,2,1)$. Each descriptor associates with two distinct modes, each with its own energy, period, and motion. Figure 16 illustrates these four modes. For each mode, the orbital and spin motions are initially in phase $(p=1)$, and the rocking number $n=2$ is given by the number of times that the sign of $\theta$ changes from negative to positive during each period (Sec. IV). In addition to the differences between their trajectories seen in Figs. 16(a) and 16(b), the two $(7,2,1)$ modes differ in the number of times that the sign of $\phi$ changes from negative to positive during each period, with this sign changing 3 times for the low-energy mode [Fig. 16(a)] and 


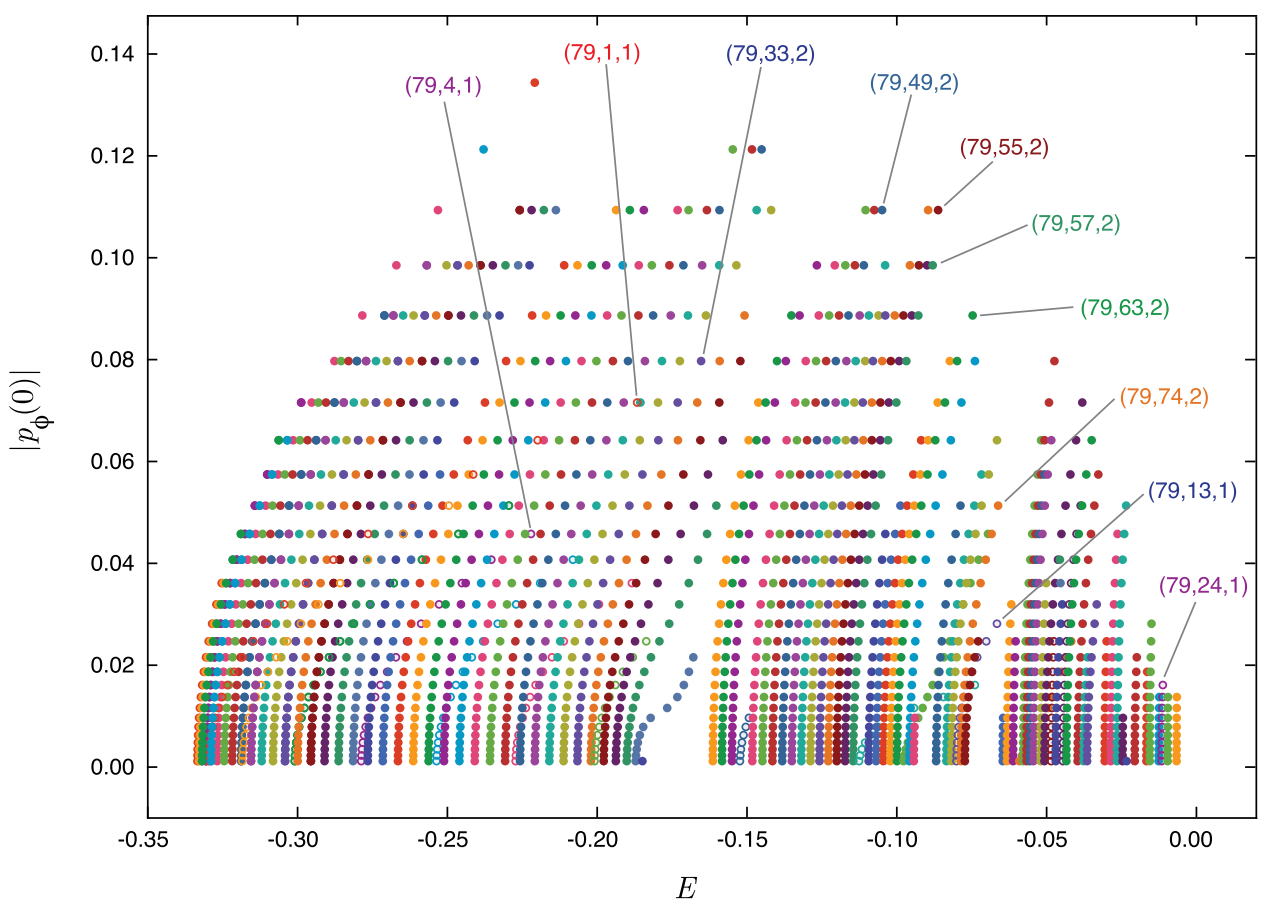

FIG. 17. Initial spin momentum $\left|p_{\phi}(0)\right|$ vs energy $E$ for finite-amplitude out-of-phase periodic states $(p=2$, filled circles) and in-phase periodic states $(p=1$, open circles) with bouncing number $m=79$ and various rocking numbers $n$. States illustrated in Figs. (18) and (19) are labeled by their mode descriptors $(m, n, p)$.

changing 16 times for the high-energy mode [Fig. 16(b)]. In addition to the differences between their trajectories seen in Figs. 16(c) and $16(\mathrm{~d})$, the two $(11,2,1)$ modes differ in a similar way, with the sign of $\phi$ changing between 2 and 9 times for the low-energy mode, depending on the initial spin angular momentum [Fig. 16(c)], and changing 13 times for the high-energy mode [Fig. 16(d)]. For more details, see MagPhyx demos 48-51. ${ }^{2}$

Irreducible modes with nonzero angular motion and odd bouncing numbers $m$ have single-passage trajectories, and those with even bouncing numbers have double-passage trajectories. This

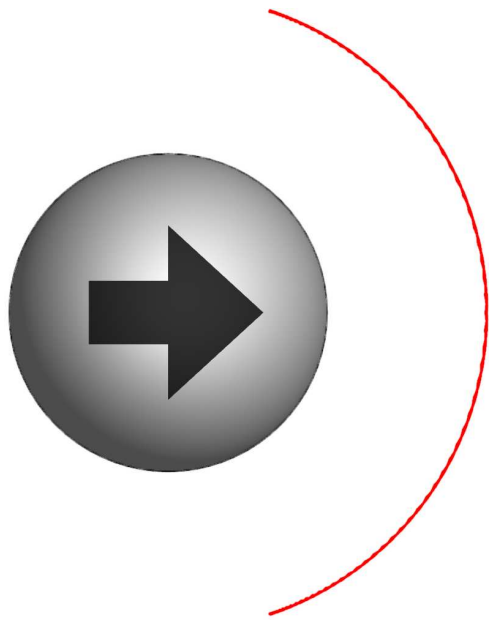

(a)

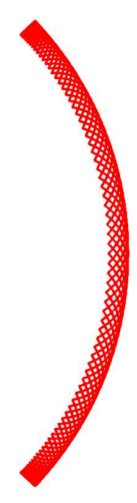

(b)

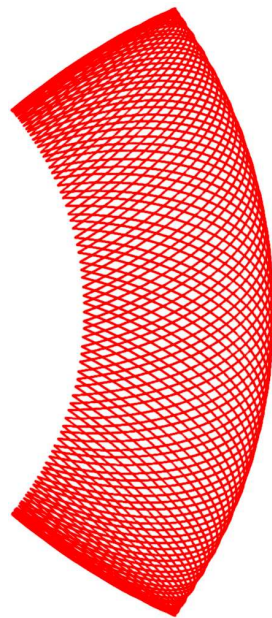

(c)

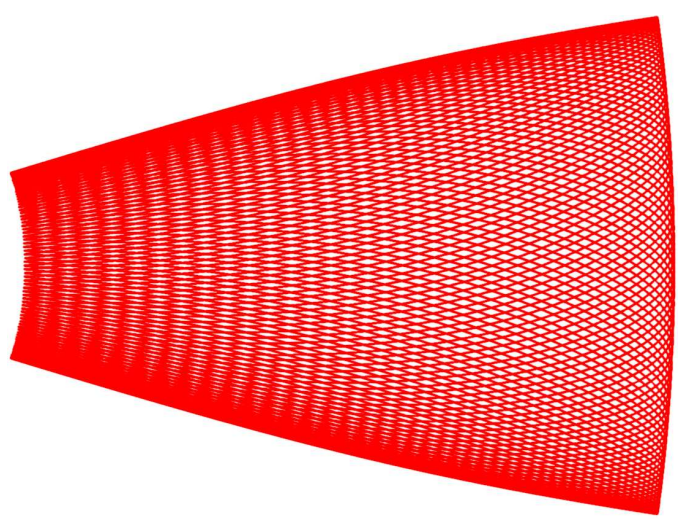

(d)

FIG. 18. Single-passage in-phase MagPhyx trajectories for modes $(79,1,1),(79,4,1),(79,13,1)$, and $(79,24,1)$, shown, respectively, in panels (a)-(d). The length scales for all four trajectories are the same and are set by the size of the gray (fixed) sphere. 


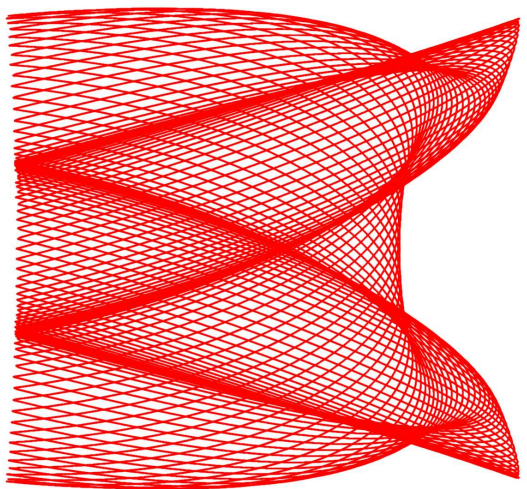

(a)

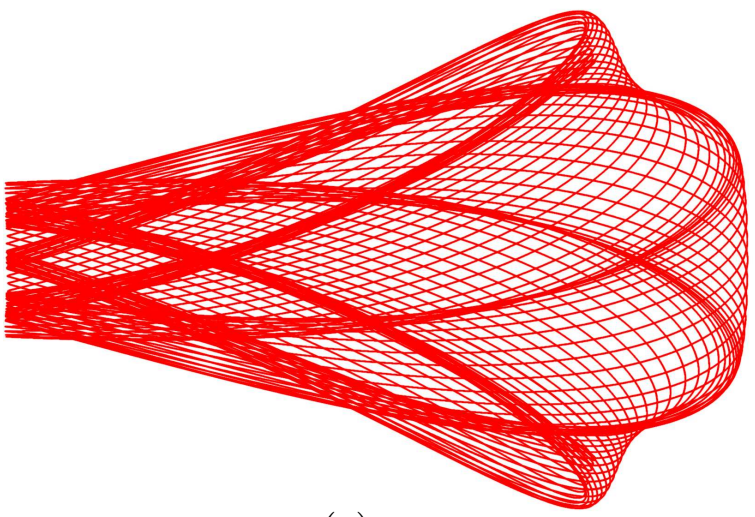

(c)

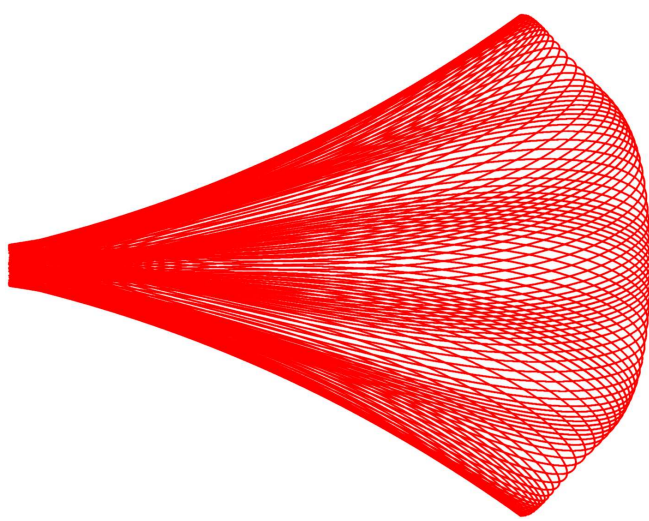

(e)

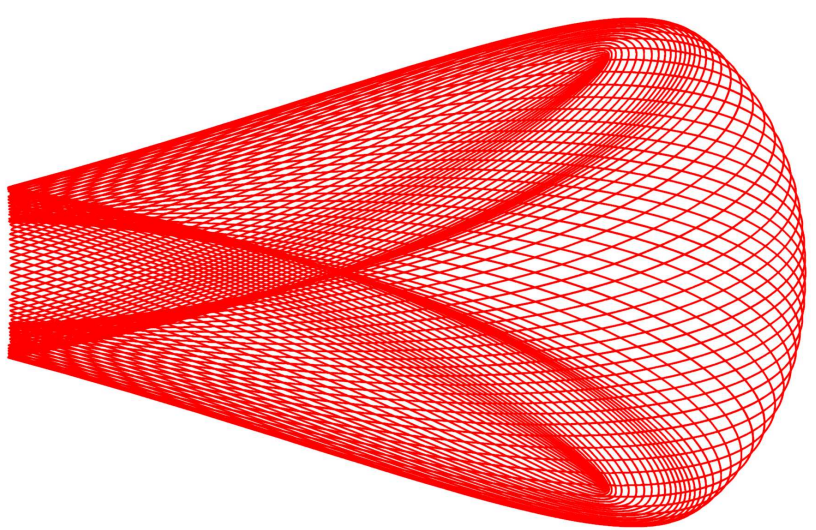

(b)

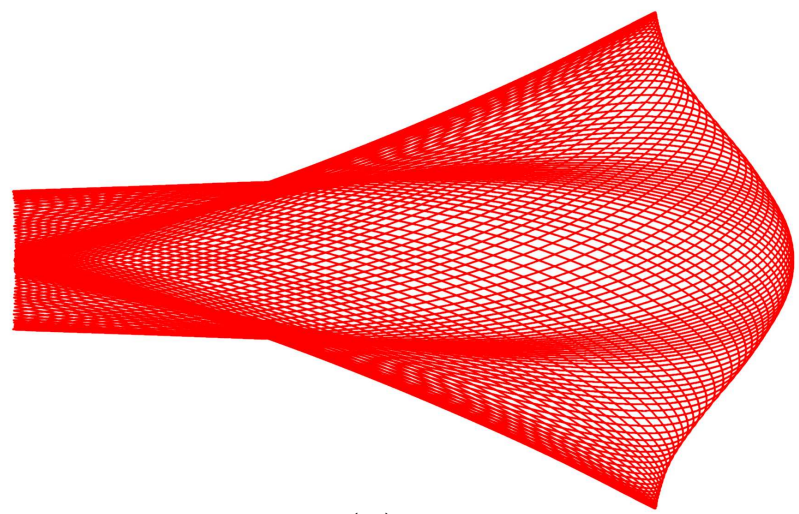

(d)

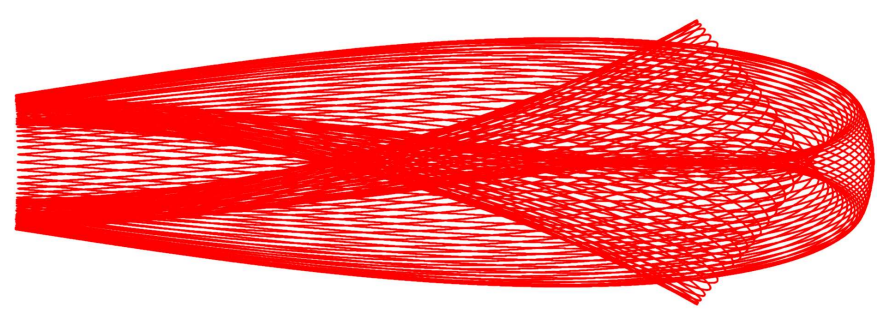

(f)

FIG. 19. Single-passage out-of-phase MagPhyx trajectories for modes $(79,33,2)$ "the owl" (a), (79,49,2) "the alien" (b), $(79,55,2)$ "the vase" (c), (79,57,2) "the stingray" (d), $(79,63,2)$ "the fan" (e), and $(79,74,2)$ "the cigar" (f). See Table II for information on freely available online MagPhyx visualizations of the time-development of these modes. 
is a consequence of the symmetry of the initial conditions and of the hard-sphere collisions: The free sphere starts at $(r, \theta, \phi)=(1,0,0)$, just after leaving contact with the fixed sphere, with nonzero $p_{\theta}(0)$ and $p_{\phi}(0)$. Consequently, the free sphere is launched at a nonzero angle $\alpha$ relative to the $+x$ axis. For the trajectory to be periodic, the final collision must be at the starting point $(r, \theta, \phi)=(1,0,0)$, at the end of one period of the motion. This collision is a hardsphere collision, so the angle of approach to the final collision must be $-\alpha$. Thus, for $m=1$, the free sphere cannot retrace its steps as it completes one period and must return to its starting point along a different path. For $m=3$, the sphere must bounce once in the first quadrant and once in the fourth quadrant before returning to its initial position for the final bounce, and so on for $m=5,7, \ldots$. For $m=2$, the free sphere must retrace its steps so that the first collision, at time $t=T / 2$, occurs at $(r, \theta, \phi)=(1,0,0)$, so that it can return to this position at time $t=T$. For $m=4$, the sphere must bounce two more times, once in the first quadrant and once in the fourth quadrant, and so on for $m=6,8, \ldots$.

To illustrate the richness and variety of our periodic finiteamplitude trajectories, we consider bouncing number $m=79$. Figure 17 shows $\left|p_{\phi}(0)\right|$ vs $E$ for the 13 in-phase modes and 93 out-of-phase modes found for $m=79$, all of which are irreducible (Table I).

Figure 18 shows single-passage trajectories for four of these inphase modes, which resemble Spirograph paths. ${ }^{26}$ Panel (a) shows mode $(79,1,1)$, for which the free sphere bounces 79 times while completing one cycle of orbital oscillation, starting at $\theta=0$ initially and passing in turn through the values $\theta=71^{\circ}, 0,-71^{\circ}$, and 0 at times $t=T / 4, T / 2,3 T / 4$, and $T$ to complete one period of the motion. The free sphere remains very close to the fixed sphere and collides with it frequently, giving a short overall period, $T=20.06$ (Table II). At first glance, the trajectory looks like a circular arc subtending an angle of $142^{\circ}$, but at screen resolutions, one can just discern a subtle scalloped substructure, with 79 scallops corresponding to the 79 bounces. Panel (b) shows mode $(79,4,1)$, for which the free sphere bounces 79 times during four cycles of orbital oscillation. The amplitude of the radial motion is larger and the collisions are less frequent than for $(79,1,1)$, giving an easily discerned substructure and a longer overall period $T=67.24$ (Table II). Modes $(79,13,1)$ and $(79,24,1)$, shown in panels (c) and (d), have yet greater radial amplitudes, less frequent collisions, and longer periods.

Shown in Fig. 19 are trajectories for six out-of-phase modes for $m=79$. In contrast with the in-phase modes, which have trajectories that are similar except for their radial and orbital amplitudes, the trajectories for the out-of-phase modes show great variety. The six modes shown represent a small fraction of the possible trajectories and were selected to illustrate their folded textures, complex symmetries, and breathtaking beauty. The time-development of these trajectories is particularly fascinating; the symmetry of the final trajectory sometimes emerges only in the late stages of the motion, and the order of appearance of the various traces that make up the whole seems virtually impossible to predict. Yet each of these trajectories is a single, continuous, closed path that crosses over itself many times before meeting up with its starting point to complete one period $T$ of the motion, and retraces its steps during each subsequent period

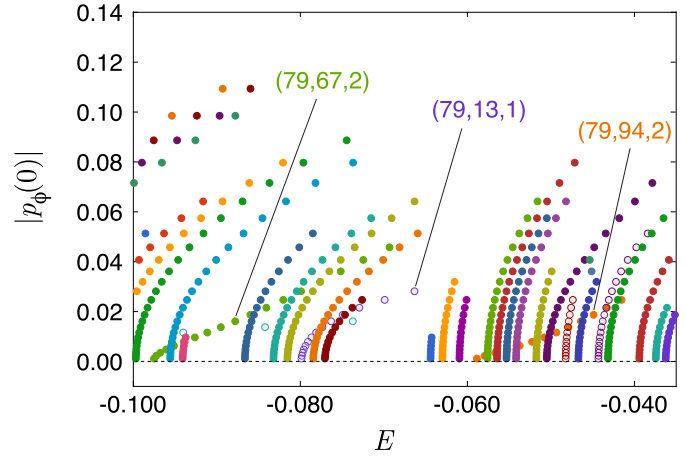

FIG. 20. Closeup of high-energy finite-amplitude states from Fig. 17 showing initial spin momentum $\left|p_{\phi}(0)\right|$ vs energy $E$ with bouncing number $m=79$.

of the motion. See Table II for information on freely available online MagPhyx visualizations of the time-development of these states.

Figures 7,12 , and 17 indicate that the threshold energy $E_{m, 1,2}$ decreases with increasing $m$, with $E_{m, 1,2} \rightarrow 0$ as $m \rightarrow \infty$. This limit satisfies $m \gg n$, the condition for small-amplitude modes, for which Eqs. (28) and (29) are valid. In this limit, the amplitudes of

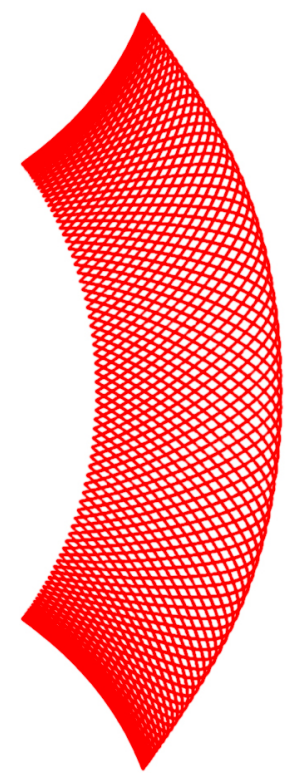

(a)

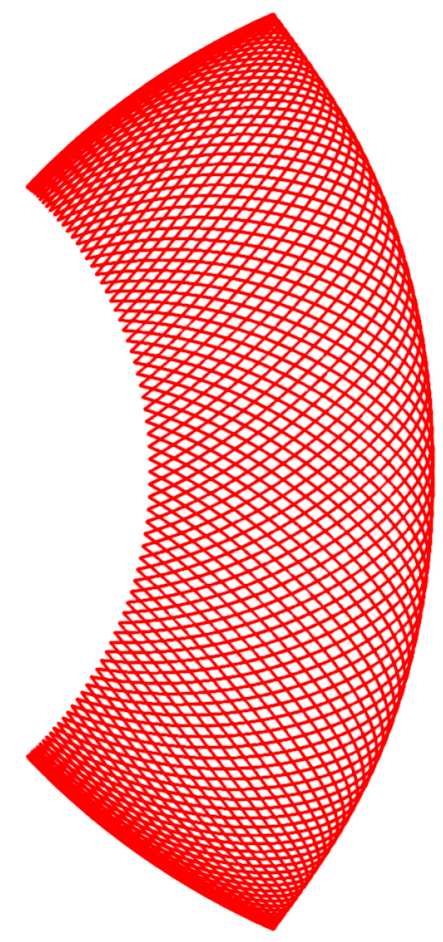

(b)
FIG. 21. Single-passage out-of-phase MagPhyx trajectories for modes $(79,67,2)$ (a) and $(79,94,2)(b)$. 
the radial, orbital, and spin motions are all infinitesimal, the free sphere remains very near its stable equilibrium position, and collides frequently with the fixed sphere.

Figure 20 is a closeup of a region of Fig. 17 with intersecting mode traces. Particularly, the $p_{\phi}(0)$ vs $E$ traces for modes $(79,67,2)$, $(79,13,1)$, and $(79,94,2)$ intersect traces for several other modes. These intersections emphasize that points in plots of $p_{\phi}(0)$ vs $E$ do not uniquely specify the initial conditions and illustrate the variety of the shapes of the traces.

As discussed in Sec. IV, we use the initial position $r(0)=1$, $\theta(0)=0$, and $\phi(0)=0$ for all of our simulations and seek periodic solutions for various $p_{r}(0)>0, p_{\theta}(0)$, and $p_{\phi}(0)$. Given that these three initial momenta are related to the energy $E$ through Eq. (35), the initial conditions may be specified either by specifying values of these three initial momenta or by specifying two of these momenta and the energy. Thus, in addition to values for $p_{\phi}(0)$ and $E$, one other initial momentum, $p_{\theta}(0)$ or $p_{r}(0)$, must be specified in order to completely specify the initial conditions. Thus, at intersections between mode traces in Fig. 20, values of $p_{\phi}(0)$ and $E$ for the two traces are the same, but values of $p_{\theta}(0), p_{r}(0)$, and $n$ are not, ensuring that the two modes are distinct.

Shapes of mode traces vary widely and include variations in the slope of $p_{\phi}(0)$ vs $E$ as $p_{\phi}(0) \rightarrow 0$. Along each mode trace, the three initial momenta depend on $E$, and no simple relationship exists between $p_{\phi}(0)$ and $E$, though the three initial momenta must obey Eq. (35).

\section{CONCLUSIONS}

We investigate the periodic motion of a free uniformly magnetized sphere subject to the fields of a second, fixed sphere, when the free sphere is located initially at its stable equilibrium point, $(r, \theta, \phi)=(1,0,0)$. We show that these initial conditions produce a rich spectrum of symmetric periodic modes. Taking a cue from our small-amplitude analysis, we characterize these modes by their bouncing numbers $m$, rocking numbers $n$, and phases $p$.

We find 1243 distinct periodic modes numerically, each with a threshold energy $E_{m n p}$ and an associated period $T_{m n p}$, and each with a family of states with differing amplitudes of angular motion. One of these is mode $(m, n, p)=(157,580,2)$, with $T_{m n p}=11298$, which requires about 16000 Runge-Kutta time steps to integrate. MagPhyx visualizations of the modes that are illustrated in this paper are freely available online (Table II). In Paper II, we investigate scaling relationships for $E_{m n p}$ and $T_{m n p} .{ }^{18}$

We show that irreducible modes with angular motion and odd bouncing numbers $m$ traverse their trajectories just once per period and show that irreducible modes with nonzero angular motion and even bouncing numbers traverse their trajectories twice during one period of the motion, once in each direction.

Of interest are the stability of the 1243 modes. We have noticed that, when our simulations are allowed to run over several periods, the trajectory eventually diverges from the periodic trajectory for some modes, presumed unstable, and remains on track for other modes, presumed stable. We would like to compute Lyapunov exponents for the various modes to discover which modes are stable and to attempt to discover why. We would also like to characterize the initial conditions generally resulting in periodic, quasiperiodic, and chaotic motion of the system. We are particularly interested in investigating the behavior of the free sphere when located initially at the unstable equilibrium point $(r, \theta, \phi)=(1, \pi / 2, \pi)$.

Our search for periodic states excludes high-energy periodic states for which the free sphere circumnavigates the fixed sphere. We have found such periodic states when the free sphere is constrained to remain in frictionless contact with the fixed sphere at all times. ${ }^{14} \mathrm{We}$ expect such states to exist for bouncing modes, and we are interested in searching for them.

Whether the structure of our periodic modes appears in other dynamical systems is an open question. To this end, we are interested in using our bifurcation approach ${ }^{18}$ to study the gravitational billiard problem with a parabolic surface. ${ }^{17}$ This problem seems to be a good candidate because it has a symmetric periodic mode (in which the ball bounces vertically from the center of the parabola) that seems similar to the radial bouncing mode from which all of our modes bifurcate (Sec. I).

\section{REFERENCES}

${ }^{1} \mathrm{~S}$. Qu, The Zen gallery, see http://zenmagnets.com/gallery/ (last accessed May 20, 2019).

${ }^{2}$ B. F. Edwards, Educational value of neodymium magnet spheres: Expert report in the matter of Zen magnets, LLC, CPSC Docket No. 12-2, Appendix D, 10/20/2014 (Redacted), see https://drive.google.com/file/d/0Bw7DdocNZGQgW ThTb3VvUHYza2s/view (last accessed May 20, 2019).

${ }^{3}$ B. F. Edwards, Educational value of neodymium magnet spheres: Expert report in the matter of Zen magnets, LLC, CPSC Docket No. 12-2, 10/20/2014 (Redacted), see https://drive.google.com/file/d/0Bw7DdocNZGQgWThTb3VvU HYza2s/view (last accessed May 20, 2019).

${ }^{4} \mathrm{~J}$. Weis and D. Levesque, "Chain formation in low density dipolar hard spheres: A Monte Carlo study,” Phys. Rev. Lett. 71(17), 2729 (1993).

${ }^{5} \mathrm{~A}$. Clarke and G. Patey, "Ground state configurations of model molecular clusters,” J. Chem. Phys. 100(3), 2213-2219 (1994).

${ }^{6}$ R. Messina, L. A. Khalil, and I. Stanković, "Self-assembly of magnetic balls: From chains to tubes,” Phys. Rev. E 89(1), 011202 (2014).

${ }^{7}$ C. L. Hall, D. Vella, and A. Goriely, "The mechanics of a chain or ring of spherical magnets," SIAM J. Appl. Math. 73(6), 2029-2054 (2013).

${ }^{8}$ D. Vella, E. du Pontavice, C. L. Hall, and A. Goriely, "The magneto-elastica: From self-buckling to self-assembly," Proc. R. Soc. A Math. Phys. Eng. Sci. 470(2162), 20130609 (2014).

${ }^{9}$ N. Vandewalle and S. Dorbolo, "Magnetic ghosts and monopoles," New J. Phys. 16(1), 013050 (2014).

${ }^{10}$ J. Boisson, C. Rouby, J. Lee, and O. Doaré, "Dynamics of a chain of permanent magnets," EPL 109(3), 34002 (2015).

${ }^{11}$ J. Schönke and E. Fried, "Stability of vertical magnetic chains," Proc. R. Soc. A Math. Phys. Eng. Sci. 473(2198), 20160703 (2017).

${ }^{12}$ G. L. Pollack and D. R. Stump, "Two magnets oscillating in each other's fields," Can. J. Phys. 75(5), 313-324 (1997).

${ }^{13}$ B. F. Edwards, D. M. Riffe, J. Ji, and W. A. Booth, "Interactions between uniformly magnetized spheres," Am. J. Phys. 85(2), 130-134 (2017).

${ }^{14}$ B. F. Edwards and J. M. Edwards, "Periodic nonlinear sliding modes for two uniformly magnetized spheres," Chaos 27(5), 053107 (2017).

${ }^{15} \mathrm{P}$. Haugen and B. Edwards, "Dynamics of two freely rotating dipoles," Am. J. Phys. (in press) (2020)

${ }^{16}$ H. Lehtihet and B. Miller, "Numerical study of a billiard in a gravitational field," Phys. D Nonlinear Phenom. 21(1), 93-104 (1986).

${ }^{17}$ H. Korsch and J. Lang, “A new integrable gravitational billiard," J. Phys. A Math. Gen. 24(1), 45 (1991).

${ }^{18}$ B. F. Edwards, B. A. Johnson, and J. M. Edwards, "Periodic bouncing modes for two uniformly magnetized spheres. II. Scaling," Chaos 30, 013131 (2020). 
${ }^{19}$ B. F. Edwards and J. M. Edwards, "Dynamical interactions between two uniformly magnetized spheres," Eur. J. Phys. 38(1), 015205 (2016).

${ }^{20}$ J. Edwards, MagPhyx visualization software, see http://edwardsjohnmartin. github.io/MagPhyx/ (last accessed June 12, 2019).

${ }^{21}$ J. A. Nelder and R. Mead, "A simplex method for function minimization," Comput. J. 7(4), 308-313 (1965).

${ }^{22}$ B. Gough, GNU Scientific Library Reference Manual, 3rd ed. (Network Theory Ltd., 2009).
${ }^{23}$ See github.com/edwardsjohnmartin/MagPhyx for source code of the MagPhyx software.

${ }^{24}$ See edwardsjohnmartin.github.io/MagPhyxP/vis for the state and spider plot visualizations.

${ }^{25}$ See github.com/edwardsjohnmartin/MagPhyxP for C++ source code for magnet path tracing.

${ }^{26}$ D. Fisher, Wikipedia: Spirograph, see https://en.wikipedia.org/wiki/Spirograph (last accessed July 25, 2019). 